\title{
Stop signals delay synchrony more for finger tapping than vocalization: A dual modality study of rhythmic synchronization in the stop signal task
}

\author{
Leidy J. Castro-Meneses ${ }^{\text {Corresp., }}{ }^{1,2,3}$ ， Paul F. Sowman ${ }^{1,2}$ \\ 1 Perception in Action Research Centre (PARC), Department of Cognitive Science, Macquarie University, North Ryde, NSW, Australia \\ 2 Australian Research Council Centre of Excellence in Cognition and its Disorders (CCD), Macquarie University, North Ryde, NSW, Australia \\ 3 The MARCS Institute for Brain, Behaviour and Development, University of Western Sydney, Bankstown, Australia \\ Corresponding Author: Leidy J. Castro-Meneses \\ Email address: I.castro-meneses@westernsydney.edu.au
}

Background. A robust feature of sensorimotor synchronization performance in finger tapping to an auditory pacing signal is the negative asynchrony of the tap with respect to the pacing signal. The Paillard-Fraisse hypothesis suggests that that negative asynchrony is a result of inter-modal integration, in which the brain compares sensory information across two modalities (auditory and tactile). The current study compared the asynchronies of vocalizations and finger tapping in time to an auditory pacing signal. Our first hypothesis was that vocalizations have less negative asynchrony compared to finger tapping due to the requirement for sensory integration within only a single (auditory) modality (intra-modal integration). However, due to the different measurements for vocalizations and finger responses, interpreting the comparison between these two response modalities is problematic. To address this problem, we included stop signals in the synchronization task. The rationale for this manipulation was that stop signals would perturb synchronization more in the inter-modal compared to the intra-modal task. We hypothesized that the inclusion of stop signals induce proactive inhibition, which reduces negative asynchrony. We further hypothesized that any reduction in negative asynchrony occurs in lesser degree for vocalization than for finger tapping.

Method. Thirty participants took part in this study. We compared sensorimotor synchronization in a single sensory modality (vocalizations [or auditory] to auditory pacing signal) to a dual sensory modality (fingers [or tactile] to auditory pacing signal). The task was combined with a stop signal task in which stop signals were relevant in some blocks and irrelevant in others. Response-to-pacing signal asynchronies and stop signal reaction times were compared across modalities and across the two types of stop signal blocks.

Results. In the blocks where stopping was irrelevant, we found that vocalization (-61.47 ms) was more synchronous with the auditory pacing signal compared to finger tapping (-128.29 ms). In the blocks where stopping was relevant, stop signals induced proactive inhibition, shifting the response times later. However, proactive inhibition ( $26.11 \mathrm{~ms}$ ) was less evident for vocalizations compared to finger tapping (58.06 ms).

Discussion. These results support the interpretation that relatively large negative asynchrony in finger tapping is a consequence of inter-modal integration, whereas smaller asynchrony is associated with intra-modal integration. This study also supports the interpretation that intra-modal integration is more sensitive to synchronization discrepancies compared to inter-modal integration. 
1

$21{ }^{a}$ Perception in Action Research Centre (PARC), Department of Cognitive Science, Macquarie 22 University, North Ryde, NSW, Australia.

23 b Australian Research Council Centre of Excellence in Cognition and its Disorders (CCD), 24 Department of Cognitive Science, Macquarie University, North Ryde, NSW, Australia.

25 c The MARCS Institute for Brain, Behaviour and Development, Western Sydney University, 26 NSW, Australia.

$27 *$ Correspondence: 1.castro-meneses@westernsydney.edu.au 


\subsection{Abstract}

Background. A robust feature of sensorimotor synchronization performance in finger tapping to an auditory pacing signal is the negative asynchrony of the tap with respect to the pacing signal. The Paillard-Fraisse hypothesis suggests that that negative asynchrony is a result of inter-modal integration, in which the brain compares sensory information across two modalities (auditory and tactile). The current study compared the asynchronies of vocalizations and finger tapping in time to an auditory pacing signal. Our first hypothesis was that vocalizations have less negative asynchrony compared to finger tapping due to the requirement for sensory integration within only a single (auditory) modality (intra-modal integration). However, due to the different measurements for vocalizations and finger responses, interpreting the comparison between these two response modalities is problematic. To address this problem, we included stop signals in the synchronization task. The rationale for this manipulation was that stop signals would perturb synchronization more in the inter-modal compared to the intra-modal task. We hypothesized that the inclusion of stop signals induce proactive inhibition, which reduces negative asynchrony. We further hypothesized that any reduction in negative asynchrony occurs in lesser degree for vocalization than for finger tapping.

Method. Thirty participants took part in this study. We compared sensorimotor synchronization in a single sensory modality (vocalizations [or auditory] to auditory pacing signal) to a dual sensory modality (fingers [or tactile] to auditory pacing signal). The task was combined with a stop signal task in which stop signals were relevant in some blocks and irrelevant in others. Response-to-pacing signal asynchronies and stop signal reaction times were compared across modalities and across the two types of stop signal blocks.

Results. In the blocks where stopping was irrelevant, we found that vocalization (-61.47 ms) was more synchronous with the auditory pacing signal compared to finger tapping (-128.29 ms). In the blocks where stopping was relevant, stop signals induced proactive inhibition, shifting the response times later. However, proactive inhibition $(26.11 \mathrm{~ms})$ was less evident for vocalizations compared to finger tapping (58.06 ms). 
58 Discussion. These results support the interpretation that relatively large negative asynchrony in

59 finger tapping is a consequence of inter-modal integration, whereas smaller asynchrony is

60 associated with intra-modal integration. This study also supports the interpretation that intra-

61 modal integration is more sensitive to synchronization discrepancies compared to inter-modal

62 integration. 
63

64 65 66

\subsection{Introduction}

The sensorimotor synchronization (SMS) task is used to study the ability of humans to coordinate their movements in time with an external beat. The most common experimental conceptualization of this task is finger tapping, whereby participants tap with a finger in time to an auditory pacing stimulus (Repp, 2005). Performance on this task is a useful index of rhythmic timing ability and has been used to investigate, amongst other things, disorders in which timing is thought to be impaired (e.g., Carroll, O’Donnell, Shekhar, \& Hetrick, 2009; Falk, Müller, \& Dalla Bella, 2015; Rubia, Taylor, Taylor, \& Sergeant, 1999), the plasticity of timing that occurs with musical training (Aschersleben, 2002; Repp, 2005) and to study multiple timing mechanisms (Iversen \& Balasubramaniam, 2016; Studenka, Zelaznik, \& Balasubramaniam, 2012). The finger-tapping task gives rise to two main metrics that index rhythmic timing performance. The first relates to the accuracy of tapping in relation to the timing of the pacing rhythm and is usually termed the 'asynchrony'. The second measure obtained from this task is the variability of inter-tap intervals, which refers to how regular taps are, relative to the pacing stimulus (Chen, Ding, \& Scott Kelso, 2001; Iversen \& Balasubramaniam, 2016; Studenka et al., 2012).

An interesting phenomenon that is observed during finger tapping to auditory pacing signal, is that humans tap, not at the point of absolute temporal coincidence between tap and auditory pacing stimulus, but at about 20 to $100 \mathrm{~ms}$ prior to the auditory pacing signal (see e.g., Aschersleben, 2002; Sugano, Keetels, \& Vroomen, 2012). This anticipatory relationship is referred to as 'negative asynchrony', first described more than 100 years ago (Dunlap, 1910; Johnson, 1899; Wallin, 1904). The mechanism that gives rise to this synchronization error is not agreed upon, and may well turn out to be multi-factorial.

There are a number of explanations for the negative mean asynchrony (hereafter NMA). These explanations may be competing but not mutually exclusive (see Aschersleben, 2002; Repp, 2005; Repp \& Su, 2013 for reviews). The transmission hypothesis, also called the "Paillard-Fraisse" hypothesis, proposed by Fraisse in 1980 and who credited it to Paillard (cited in Aschersleben \& Prinz, 1995) argues that the NMA comes for different conduction times between a sensory channel and the brain. Another explanation of the NMA is the sensory accumulator hypothesis 
94 (Aschersleben, 2002) that argues that the NMA is a result of a central representation of the 95 average of various sensory channels processed at different rates. Unlike a peripheral coding of 96 sensory information in the Paillard-Fraisse hypothesis, the sensory accumulator hypothesis

97 proposes an overall central coding of sensory information.

99 There are other theories that explain the NMA from a biomechanical perspective. For example,

100 the optimal selection hypothesis (Vaughan, Rosenbaum, Diedrich, \& Moore, 1996) argues that in

101 finger tapping, the energy used is minimized while satisfying overt task demands by

102 simultaneously selecting one or two optimal limb resonant frequencies that match the task

103 (driving) frequency. Unlike the two previous hypotheses that explained NMA as a function of

104 the transmission times of sensory channels, the optimal selection hypothesis explains NMA as a

105 function of the optimal effector's resonant frequency. See also Pikovsky, Rosenblum, and Kurths

106 (2003) that give a more holistic overview of how tapping can be regarded as an oscillatory motor

107 activity that is coupled to an external driving oscillator (i.e., the pacing signal. See Strogatz, 2003

108 for a book review). In a similar fashion, Hove, Fairhurst, Kotz, and Keller (2013) found that

109 certain pacing stimuli produce better synchronization than others in finger tapping. Specifically,

110 beeps are more accurately synchronized to than continuous sirens and, that moving stimuli are

111 more accurately synchronized to than static flashes. The explanation of these phenomena begets

112 the 'modality appropriateness' theory, which suggests that auditory and visual systems may have

113 a greater capacity for encoding certain stimuli than others.

115 Another explanation for the NMA is the linear phase correction theory (Schulze \& Vorberg,

116 2002) that explains synchronization via a statistical argument in which participants try to

117 minimise variance of their asynchronies through a linear phase correction. See also Large and

118 Jones (1999) for a description of other statistical theories.

120 In a slightly different proposal of how synchronization occur, Large and Jones (1999) suggest the

121 'attentional dynamics' theory. This theory postulates two entities: external rhythms, which are

122 created by the outside events, and internal rhythms, which are generated by temporal

123 expectancies. It postulates that external and internal rhythms are coordinated via entrainment. 
125 The present study presents evidence in favour of the Paillard-Fraisee hypothesis. Thus, in the

126 following we explain this account in more detail. This hypothesis suggests that sensory

127 transduction and afferent conduction times play a crucial role in SMS. Sensory transduction

128 refers to the conversion, by a sensory receptor, of a physical stimulus into an electrical potential

129 that is then able to be transmitted to the central nervous system (Lumpkin \& Caterina, 2007).

130 Afferent conduction time, on the other hand, refers to the time that elapses as information is

131 carried from a sensory neuron to the central nervous system.

133 In the context of finger tapping to an auditory pacing signal, the Paillard-Fraisee hypothesis

134 explains that the NMA occurs because the brain is synchronizing to two sensory modalities: the

135 tactile/kinaesthetic sensory modality and the auditory modality. Given that the

136 tactile/kinaesthetic modality takes a longer time to reach the brain due to differences in sensory

137 transduction and afferent conduction times compared to the auditory sensory modality,

138 coincident stimulation of both sensory channels would give rise to asynchronous registration in

139 the brain. The consequence of this is that tapping to an auditory pacing signal can be perceived to

140 be 'in time', and not ahead of the beat as is the veridical case.

142 Example evidence supporting the Paillard-Fraisee hypothesis comes from an experiment where

143 participants were informed of the size and direction of their asynchronies, they were

144 subsequently able to tap with greatly reduced NMA, reporting a subjective experience of having 145 to delay their responses (Aschersleben, 2002, pp. 67-68). In another study, when participants

146 received a delayed feedback after their taps, the asynchronies became more negative than for a

147 no-delayed feedback condition (Sugano et al., 2012). This finding indicates that participants tried

148 to recalibrate their taps to their perception of temporal coincidence. This temporal recalibration

149 has also been observed in delayed visual feedback (Cunningham, Billock, \& Tsou, 2001;

150 Stetson, Cui, Montague, \& Eagleman, 2006) and tactile motor recalibration (Heron, Hanson, \&

151 Whitaker, 2009).

152

153 More evidence supporting the Paillard-Fraisee hypothesis comes from an experiment showing

154 that foot tapping, as opposed to finger tapping, to an auditory pacing signal exhibits a larger

155 negative asynchrony, possibly because the tactile afferent conduction time of the foot is longer 
156 compared to that of the hand (Aschersleben \& Prinz, 1995; Billon, Bard, Fleury, Blouin, \&

157 Teasdale, 1996).

158

159 Müller et al. (2008) compared tactile and auditory pacing signals in finger and toe tapping tasks.

160 They found that negative asynchrony was significantly smaller for both finger and toe tapping

161 when the pacing signal was tactile compared to auditory. While finger and toe tapping to a tactile

162 pacing signal had a NMA of approximately $8 \mathrm{~ms}$ (not statistically different from the onset of the

163 tactile pacing signal), finger and toe tapping to an auditory pacing signal exhibited a NMA of

164 approximately $40 \mathrm{~ms}$ that was significantly negative with reference to the onset of the auditory

165 pacing signal. The authors interpreted this finding as evidence to support the argument that SMS

166 is affected by whether sensory integration is inter- or intra- modal. Because the sensory feedback

167 generated by finger and toe tapping occur within the same sensory modality as the tactile pacing

168 signal (i.e., intra-modal integration), the signals required for synchronization are more

169 temporally coincident centrally (in the brain). In contrast, finger and toe tapping to an auditory

170 pacing signal requires integration across two sensory modalities (i.e., inter-modal integration)

171 that have different sensory transduction and conduction times. This difference causes a temporal

172 offset centrally that results in a large NMA.

173

174 It remains an open question as to whether the findings introduced above describe a general rule

175 regarding the synchronization consequences determined by intra- versus inter- modal sensory

176 integration contexts, as to date, the descriptions of this phenomenon are limited to the

177 synchronization of effectors controlled by the corticospinal system (like the finger or toe) to a

178 tactile or auditory pacing signals. Therefore, in order to advance this line of research we

179 developed the current study to test SMS in another sensory/effector modality pairing, that being

180 an auditory pacing signal with a vocal response and compared this to the classic finger tapping to

181 an auditory pacing signal task. Finger tapping with auditory pacing requires the

182 tactile/kinaesthetic and auditory sensory modalities respectively. The tactile/kinaesthetic

183 modality refers to finger tapping, while the auditory modality refers to the auditory pacing signal. 184

185 On the other hand, the sensory integration required for vocalization to an auditory pacing signal 186 occurs within the same sensory modality because we assume that vocalization produces a sound, 
187 which is equivalent to the auditory feedback of experiment 2 in Aschersleben and Prinz (1995).

188 In experiment 2 of Aschersleben and Prinz (1995), finger taps elicited auditory feedback.

189 Because the auditory feedback occurred within the same sensory modality as the auditory pacing

190 signal, the authors interpreted that the relative phase of finger tapping would be modulated by

191 both inter and intra-modal integration processes. Aschersleben and Prinz (1995) found that finger

192 tapping with auditory feedback exhibited a relative reduction in NMA of $17 \mathrm{~ms}$ compared to

193 finger tapping without auditory feedback.

195 According to this previous study (Aschersleben \& Prinz, 1995), we predicted that finger tapping

196 (with no auditory feedback) to an auditory pacing signal would show a larger NMA due to the

197 requirement for inter-modal integration (i.e., differences in sensory transduction and afferent

198 conduction times). In contrast to finger tapping, in the intra-modal case, vocalizations to an

199 auditory pacing stimulus would show significantly less NMA, in accordance with Müller et al.

200 (2008) and Aschersleben and Prinz (1995).

201

202 However, a significant problem arises when attempting to test this hypothesis. Finger tapping and vocalizations are measured via two different methods. To calibrate the time constants between these two measures is unlikely to be precise. Our proposed solution to this problem was to measure and compare the responses to a perturbation of the synchronization time across response modalities. We based our perturbation on that presented in Fischer, Tan, Pogosyan, and Brown (2016) who demonstrated that synchronization time is modulated in the presence of a secondary inhibitory control task: the stop signal task (Logan \& Cowan, 1984).

210 The stop signal task is a paradigm that measures the time it takes to stop an ongoing response.

211 The experimental task consists of go and stop trials. In go trials, participants respond rapidly to a

212 go signal. Stop trials start out as go trials but following the go signal, a stop signal appears. The

213 stop signal indicates that participants should attempt to arrest their already initiated response.

214 Stop trials usually make up a minority of all trials (e.g., 25\% is a typical proportion of stop

215 trials). The speed at which participants are able to stop is indexed by the stop signal reaction time

216 or SSRT. SSRT is considered primarily a measure of reactive inhibition because participants

217 react to the stop signals by attempting to arrest their response (Aron, 2011). 
219 Another measure that can be obtained from variants of the stop signal task is proactive inhibition 220 (Chikazoe et al., 2009; Jaffard et al., 2008). This refers to how much participants prepare to stop 221 in anticipation of stop signals by slowing their go responses. To measure proactive inhibition, the 222 experimental task requires a control condition in which stopping is not required. The go RT in 223 the control condition is then compared to the go RT in the condition where stopping is required 224 (e.g., Castro-Meneses, Johnson, \& Sowman, 2015; Castro-Meneses, Johnson, \& Sowman, 2016; 225 Chikazoe et al., 2009; Jaffard et al., 2008). Generally, participants exhibit slower go RTs under 226 conditions where stopping is required, which is thought to be indicative of advanced preparation 227 for possible stop signals (Duque, Greenhouse, Labruna, \& Ivry, 2017), or proactive inhibition. 228

Castro-Meneses et al. (2016) used two types of blocks to measure proactive inhibition. In their study, some blocks contained go and stop trials; this was the relevant stop condition. Other blocks contained go and stop trials but participants were instructed to ignore the stop trials and treat them as if they were go trials. These blocks were the irrelevant stop condition. The irrelevant stop blocks served as the control condition, which go RT changes could be assessed against the relevant stop blocks (i.e., when stopping was required). It was found that when stopping was required in the relevant stop condition, participants slowed their go RTs compared to those RTs in the irrelevant stop condition.

The current experiment utilises a combination of the SST and the SMS as per Fischer et al. (2016). In accordance with the results of their study, we predicted that the anticipation of a stop signals before the pacing signal would induce proactive inhibition, delaying the synchronization response. This effect would be indexed by a reduction in the NMA. Although, slower go RTs in the stop signal task are considered to represent advanced preparation to stop (or proactive inhibition), we interpret that in a SMS with visual stop signals, a reduction of NMA also explains an advanced preparation to stop. However, alternative theories can also support this reduction as they have shown that an additional working memory task can impair temporal regularity (Maes,

246 Wanderley, \& Palmer, 2015; Repp \& Su, 2013). For example, Maes et al. (2015) shows that in a SMS with a secondary working memory task, which consisted in a concurrent digit-switch counting task, the temporal regularity was highly impaired when the cognitive load was high. 
250 Our subsequent prediction was that visual stop signals would reduce NMA to a lesser degree for vocalizations than they would for finger tapping. Our rationale for this prediction was that, in the

252 case of finger tapping to an auditory pacing signal, the inter-modal integration between the

253

254

255

256

257

258

259

260

261

262

263

264

265

266

267

268

269

270

271

272

273

274

275

276

277

278

279 tactile/kinaesthetic and auditory modalities allows more 'room' for a perturbation to shift the synchronization timing. On the contrary, when vocalizations are synchronized to an auditory pacing signal, the intra-modal integration between the auditory modality is more likely to perceive a disruption in the accurate synchronization and therefore correct it. In other words, intra-modal integration should be more sensitive to temporal coincidence detection than intermodal integration (Grondin \& Rousseau, 1991; Grondin, Roussel, Gamache, Roy, \& Ouellet, 2005; Sugano et al., 2012).

By combining the stop signal task and the SMS, Fischer et al. (2016) show that the NMA is significantly reduced when the possibility of a stop signal is imminent. Specifically, the inclusion of relevant stop signals induced a reduction in the NMA (for finger tapping). We predicted that such a shift in the NMA would be less tolerable in a vocalization task because synchronization in this case is underpinned by intra-modal integration.

In sum, the evidence presented so far suggests that when performing SMS tasks, the NMA is affected by differences in sensory transduction and afferent conduction between sensory modalities (e.g., the negative asynchrony of finger tapping to a tactile pacing signal is significantly smaller compared to that for finger tapping to an auditory pacing signal). When the tap and the pacing signal occur within the same sensory modality (thus, intra-modal integration), the NMA is reduced due to equivalent sensory transduction or afferent conduction times.

To further investigate the Paillard-Fraisse modal integration hypothesis of asynchronous tapping, we investigated finger tapping and vocalizations to test two specific hypotheses. 1: that in a control condition (i.e., where synchronization was important while stopping was irrelevant), vocalizations to auditory pacing elicit shorter NMA compared to finger tapping. 2: that in an experimental condition (i.e., where both synchronization and stopping were relevant), vocalizations to auditory pacing exhibit reduce NMA, but that this reduction occur to a lesser 
280 degree than for finger tapping to auditory pacing. This lesser reduction in vocalizations occurs

281 due to intra-modal integration that engenders more sensitive temporal coincidence detection than

282 inter-modal integration.

283

284

1.3. Methods

285

286

287

\subsubsection{Participants}

Forty-four participants completed this study (32 females) aged (mean - SD) 19.6 - 2 years. Based 288 on the requirement of successful stopping on about $50 \%$ of the trials for validity of the measures taken from the stop signal task (Logan \& Cowan, 1984), data inclusion was limited to that taken from participants with a percentage of successful stopping that fell between 40 and $60 \%$ (see the description of the stop signal task below for a full explanation). Fourteen participants did not meet this performance criterion and hence their data were excluded from the analysis. All participants were right-handed and reported no history of neurological or psychiatric conditions. Participants received course credit for their participation. The experiment was approved by Macquarie University human research ethics committee (Ref. 5201200035).

\subsubsection{Apparatus}

298

The experimental task was coded in Presentation software (version 16.1, www.neurobs.com) and delivered via a Samsung monitor (SyncMaster SA950_LS27A950, 27 inches, 1920 x 1080 pixels, $120 \mathrm{~Hz}$ refresh rate). In audio settings, the Presentation mixer mode was DirectX. The auditory pacing signal was synthesised in Audacity (version 1.34-beta, Audacity Team, Copyright (c) 2018) at $750 \mathrm{~Hz}$ and $75 \mathrm{~ms}$ in length. The auditory pacing signal was presented via Sennheiser headphones (HD 280 pro) with up to $32 \mathrm{~dB}$ ambient noise attenuation. Vocalizations were recorded ( 8 bits, 2 channels, $48 \mathrm{kHz}$ ) via an external Shure microphone (version WH20XLR) positioned $2 \mathrm{~cm}$ from each participant's mouth. The microphone signal was amplified by an ART Tube MP Pre-Amp. Vocalizations were identified via the sound response device in Presentation software, which detected a response when a sound passed a minimal threshold (set to 0.04 on a $0-1$ scale). This scale represents a percentage of the maximum sound recording level. Finger taps produced by the right index finger were recorded via a Cedrus RB830 button box. Participants were seated approximately $80 \mathrm{~cm}$ from the monitor. 


\section{Calibration of responses}

313 Vocal responses were recorded and used to verify, offline, the accuracy of the response times

314 reported by Presentation software. A Matlab script that detected a vocal response onset based on

315 the sound envelope of the vocalization showed a high level of concordance with the software

316 voice key. This analysis is located in the supplementary material.

317

318 Further to this analysis, we calibrated the response times reported by Presentation with

319 PowerLab 8/35 (https://www.adinstruments.com/products/powerlab) that measured the veridical

320 times of the stimulus and response relative to each other. We used this to adjust the two response

321 modalities, subtracting $75.83 \mathrm{~ms}$ from the button press and $67.43 \mathrm{~ms}$ from the vocal responses.

322 Please refer to Appendix A for a description and graphical representation of this calibration

323 procedure.

324

325

\subsubsection{Stop signal task and SMS}

326 This study combined two paradigms: the stop-signal task (SST; Logan, 1994; Logan \& Cowan, 327 1984) and the SMS task, (Stevens, 1886; Wing, 2002). For a graphical illustration of the stop

328 signal task and the current study's task, please see Appendix B. This combined task consisted of 329 two blocks: relevant and irrelevant stops. Both the relevant and irrelevant stop blocks included 330 both go and stop trials. In the irrelevant stop block, participants were instructed to ignore the stop 331 trials and treat them as go trials. Thus, the irrelevant stop block was very similar to the classic 332 SMS task where participants only synchronize their responses to the pacing signal. Whereas in 333 the relevant stop block, participants had to both synchronize to the pacing signal and to try to 334 withhold their synchronization response whenever a stop signal appeared.

336 In go trials, participants were asked to synchronize to an auditory pacing signal by either finger 337 tapping or vocalizing. Finger tapping consisted of pressing a response key in time with the 338 auditory pacing signal, whereas vocalizations consisted of producing the vowel sound " $\mathrm{I}$ " as it 339 would sound in the word "hit /hrt/" in time with the auditory pacing signal. 
341 When detecting vocalization onsets, it is ideal to use a low threshold for the sound key in order

342 to mitigate against delays in the onset. However, this means that there can be false onsets

343 detected by lip noises. By using vowel sounds, participants were able to respond these sounds

344 without having to open and close their lips. We also showed to the participants how Presentation

345 software detected the vocalizations (sound device settings) and indicated how a lip sound could

346 be detected as a vocal response. We instructed participants to keep their lips apart during the

347 testing and avoid lip sounds and sighs. Furthermore, because vowel sounds can be made with

348 little jaw movement they are better for EEG/MEG studies. Because we have conducted such

349 studies previously we use consistent stimuli here in order to be able to compare our results across

350 experiments (Castro-Meneses et al., 2016; Etchell, Sowman, \& Johnson, 2012).

351

352 In stop trials, participants were asked to withhold their response when they saw a stop signal.

353 The stop signal was visually presented as a red ' $\mathrm{X}$ ' (400 font, $12 \mathrm{~cm}$ height, $10.5 \mathrm{~cm}$ width, 8.5

354 visual angle and $200 \mathrm{~ms}$ in duration) on a black background. There was a minimum of 1 and a

355 maximum of 10 go trials after any stop trial. The stop signal was initially placed $200 \mathrm{~ms}$ before

356 the pacing signal and adjusted according to a dynamic stop-signal delay (SSD) staircase (for a

357 more extensive description of the stop signal task and a similar protocol see Castro-Meneses et

358 al., 2015). Each response modality had a separate SSD staircase adjusted independently, thus

359 there were two staircases, one for finger taps and one for vocalizations. The staircase adjustment

360 changed the SSD after every stop trial by approximately $30 \mathrm{~ms}$ (to the nearest multiple of $8.3 \mathrm{~ms}$

361 as dictated by the monitor refresh rate), increasing it by $30 \mathrm{~ms}$ if participants successfully

362 inhibited their previous response and decreasing it by $30 \mathrm{~ms}$ if previous inhibition was

363 unsuccessful (Logan, Schachar, \& Tannock, 1997; Osman, Kornblum, \& Meyer, 1986, 1990;

364 Verbruggen \& Logan, 2009a). This method aims to return a percentage of successful stopping of 365 approximately $50 \%$.

366

367 A block contained 80 trials, of those, 60 trials were go trials (3/4 of total trials) and 20 trials were 368 stop trials (1/4 of total trials). The inter-onset interval between the auditory pacing signal was 369 constant at $1250 \mathrm{~ms}$. This inter-onset interval allowed a sufficient gap between stop trials, such

370 that the stop signals would be perceived as clearly occurring before the auditory pacing signal.

371 This time is still within the limits of optimal synchronization performance intervals which range 
372 from about $175 \mathrm{~ms}$ to $1580 \mathrm{~ms}$ (Bolton, 1894; London, 2002). For an illustration of the trial 373 structure, see Figure 1.

374

375 Estimating asynchronies

376 Our first aim was to estimate the time at which the finger taps or vocalizations were produced

377 with respect to the onset of the pacing signal. To do this, we subtracted the time of the pacing

378 signal $\left(t_{p s}\right)$ from the time of a response $\left(t_{r}\right): t_{p s}-t_{r}$. Positive values indicate a response occurred

379 after the pacing signal, whereas negative values indicate a response occurred before the pacing

380 signal.

381

382 Estimating the perturbation of stop signals on the asynchronies: proactive inhibition

383 Our second aim was to measure the degree by which stop signals reduce the NMA in both finger

384 tapping and vocalization. This corresponds to a common measure extracted from the stop signal

385 task called proactive inhibition (see Castro-Meneses et al., 2015 for in depth description).

386 Proactive inhibition is defined as the amount of goRT slowing due to the introduction of stop

387 signals. To measure this slowing requires both a control block, where stop signals are ignored or

388 not presented, and an experimental block where stop signals are relevant; the difference in RT

389 between these two blocks is then obtained (this is refered to block-by-block analysis, for a trial-

390 by-trial analysis see Verbruggen \& Logan, 2009b). To do this, we subtracted these response

391 times from the relevant stop condition from those of the irrelevant stop condition in both finger

392 tapping and vocalizations.

393

394 Estimating reactive inhibition

395 Reactive inhibition is a measure obtained from stop signal task performance. The analysis of

396 reactive inhibition did not have any direct impact on this study's aims but was included for

397 completeness. Reactive inhibition is indexed by the stop signal reaction time (SSRT) and is

398 based on the independent horse-race model (Logan \& Cowan, 1984). We used the integration

399 method to estimate SSRT, which is considered the most robust approach for estimating SSRT

400 (Verbruggen, Chambers, \& Logan, 2013; Verbruggen \& Logan, 2009a). Using this method,

401 SSRT was calculated by subtracting the starting time of the stop process (when participants saw

402 a stop signal) from the finishing time of the stop process. The starting time of the stop process 
403 refers to the time of the stop signal delay (or SSD). The finishing time needs to be estimated. The 404 finishing time is usually estimated by integrating the go reaction time (go RT) distribution. The 405 go distribution in the current task did not contain RTs but rather go synchronized responses (or 406 go SR), thus we estimated the finishing time from the go synchronized time distribution.

407 Synchronized responses were estimated as the time a button press or vocal response occurred 408 within the trial. The distribution of the go SR was rank ordered from shortest to the longest then, 409 the $n t h$ SR was selected, where $n$ was selected by multiplying the probability of responding on 410 stop trials (or unsuccessful stopping) by the total number of go SR. The probability of 411 responding was calculated as the number of unsuccessful stops divided by the total number of 412 stop trials. SSRT was estimated by subtracting the SSD from $n$th go SR. SSRT can only be 413 estimated in the relevant stop blocks. We first calculated it across each block and then, it was 414 averaged across blocks. A graphical representation of the variables in the classic stop signal task 415 as well as in the current task are depicted in Appendix Figure A6.

\subsubsection{Experimental design}

418 This study involved one session divided into two phases: phase-1 and phase-2. At the end of 419 phase-1, participants were presented with a message saying: "You are halfway through the 420 experiment, you have 4 more blocks to go". Each phase consisted of four blocks (in total 8 421 blocks) in which two blocks were relevant stop blocks (one finger tapping and the other vocalization) and two blocks were irrelevant stop blocks (one finger tapping and the other vocalization). The four-block order (i.e. a phase) was pseudo-randomised by two conditions.

424 Firstly, a response modality could only be followed by the other response modality of the same stop block (either irrelevant or relevant stop). Secondly, a stop block could only be followed by the other stop block once the first condition was met. For example, if the first block was a relevant stop block with finger tapping then the second block had to be the same stop block (i.e., relevant stop block) with the other response modality (i.e., vocalization). Subsequently, the third and fourth blocks were irrelevant stop blocks, the third with finger taps and the fourth block with vocalizations. At the beginning of each block, instructions were presented on the screen

431 indicating what type of stop block and response modality was to follow, see Figure 2 . The 432 purpose of the phases was to ease the task complexity. 


\subsubsection{Procedure}

435 Participants were told that they had to synchronize their response in time with an auditory pacing 436 signal and to attempt to withhold this response when a stop signal was presented. They were 437 encouraged to relax as much as they could and respond with ease. We explained that both 438 synchronizing and stopping were equally important and that they would fail to stop on about $43950 \%$ of the stop trials because the experiment adjusted itself to give them easy and hard stop 440 signals according to their performance. Each participant completed at least one practice task. The 441 practice task was a much shorter version of the experimental task; it contained 8 blocks of 12 442 trials each (4 were stop trials). First, the experimenter performed the practice task as a 443 demonstration. Then, the participant was invited to do the practice task and, on completion, their 444 performance was assessed. If they had correctly synchronized to go trials, did not have more than 4455 misses and got 2 successful and 2 unsuccessful stops, we administered the main task, 446 otherwise, the practice task was repeated.

During the experimental task, the first 10 trials in each block were always go-trials (for the 449 purpose of familiarization with the rhythm). These trials were not included in any statistical 450 analyses.

451

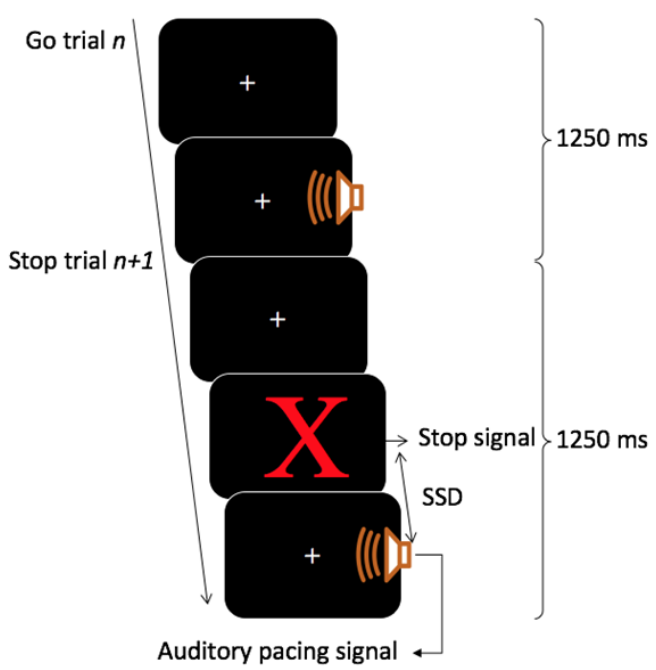

Figure 1. Trial structure. Each trial lasted for $1250 \mathrm{~ms}$. Go trials consisted of a visual fixation cross (continuously presented) and an auditory 
456

457

458

459

signal and were adjusted throughout via a staircase method in which, after a successful stop, the next stop trial was made harder by decreasing the time between the stop signals and the auditory pacing signal by $30 \mathrm{~ms}$. Contrarily, if participants were unsuccessful on a stop trial, the next stop trial was made easier by increasing the time of the stop signal in relation to the pacing signal by $30 \mathrm{~ms}$.
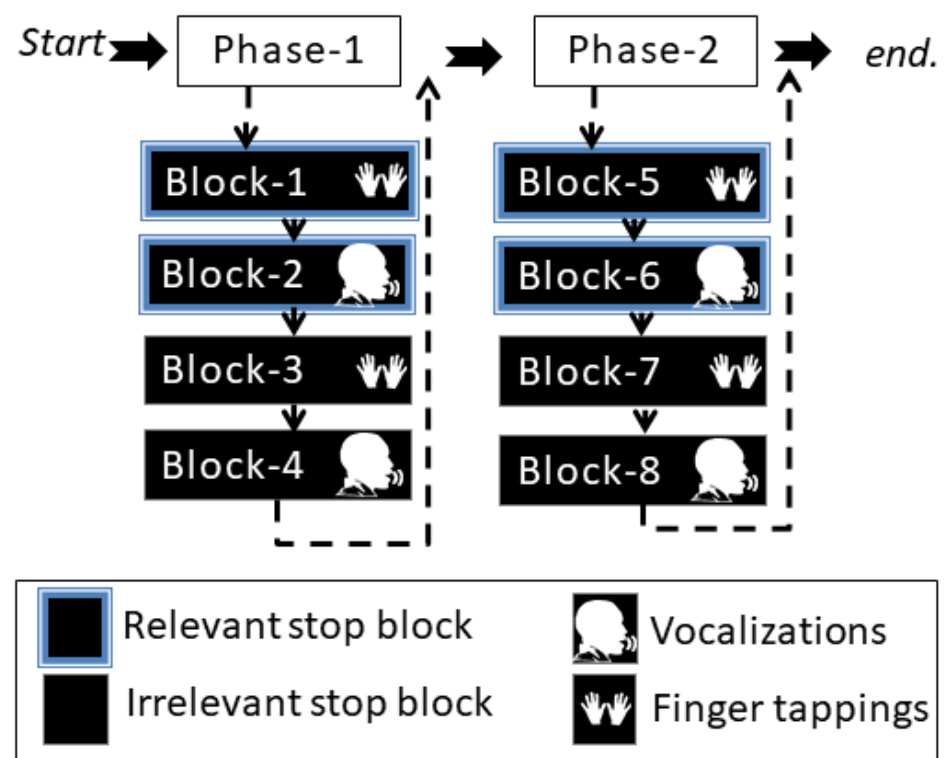

460

461

Figure 2. Illustration of the experimental design. The experimental task was divided into two phases that included four blocks. The four-block

462

order (i.e. a phase) was pseudo-randomised by the condition such that two blocks were either irrelevant or relevant stop blocks for each response

463

modality (vocalization or finger tapping).

464

465

\subsection{Results}

466

467

468

1.4.1. Asynchronies

469

$3 \times 2 \times 2 \times 2$ repeated-measures $A N O V A$

470

We first assessed to what degree the stop signals affected synchronization. To do this we

471

compared the asynchronies after every stop signal and classified them as: $1^{\text {st }}$ go after a stop

472 signal, $2^{\text {nd }}$ go after a stop signal and all other go trials (i.e., all go trials except the $1^{\text {st }}$ and $2^{\text {nd }}$ go).

473

We carried out a repeated-measures $3 \times 2 \times 2 \times 2$ ANOVA on the dependent variable: NMA,

474 with within-subject factors of 3 go types after stop signals ( $1^{\text {st }}$ go, $2^{\text {nd }}$ go and all other go), 2 stop block types (relevant stop, irrelevant stop), 2 response modalities (finger tapping, vocalization) and 2 phases (phase-1, phase-2). We were interested in the go type factor and any interaction of this factor with other factors. All results of the ANOVA are found in Appendix B, Table A1. 
477

478 The results revealed three statistically significant main effects and three interactions: stop block 479 type $\left(F(1,29)=79.31, p<0.001, \eta^{2}{ }_{p}=0.74\right)$, response modality $\left(F(1,29)=95.80, p<0.001, \eta^{2} p\right.$ $480=0.70)$, phase $\left(F(1,29)=6.20, p=0.019, \eta^{2}{ }_{p}=0.18\right)$, the two-way interaction of stop block type 481 by response modality $\left(F(1,29)=22.60, p<0.001, \eta^{2}{ }_{p}=0.44\right)$, the three-way interaction of go 482 type by stop block type by response modality $\left(F(1.7,48.5)=4.59, p=0.021, \eta^{2}{ }_{p}=0.14\right.$ -

483 Greenhouse-Geisser corrected) and the two-way interaction of go type by phase $(F(2,58)=5.88$, $\left.484 p=0.005, \eta^{2}{ }_{p}=0.17\right)$. Because we were mainly interested in the go type factor and any 485 interaction of this factor with other factors, we first describe the two significant interactions that 486 involved the go type factor. A description of the main effects and interactions that were 487 significant but did not involve the go type factor is included in Appendix C and Table A1.

Post hoc analysis following up on the two-way interaction between the go type and phase (Bonferroni corrected) revealed that the 3 go types were not significantly different within each phase $(p s>0.410)$ or across phases $(p s>0.108)$. The only significant difference was for the $1^{\text {st }}$ go type go type across phases. Specifically, the $1^{\text {st }}$ go (after a stop signal) in phase- $1(M=-65.05$ $\mathrm{ms}, S E=5.84,95 \% C I[-76.98$ to -53.12$])$ occurred $17.70 \mathrm{~ms}$ earlier than the $1^{\text {st }}$ go in phase-2 $(M=-82.75 \mathrm{~ms}, S E=6.98,95 \% C I[-97.02$ to -68.48$], p<0.001)$. All other pairwise comparisons were not statistically significant; these are shown in Appendix D, Tables A2 and A3. Because there were significant differences between the $1^{\text {st }}$ go across phases, we excluded the $1^{\text {st }}$ go after the stop signal for the subsequent analysis in the $2 \times 2 \times 2$ ANOVA.

Post hoc analyses on the three-way interaction of go type by stop block type by response modality revealed that there were no differences across the $1^{\text {st }}$ go, $2^{\text {nd }}$ go and all other go in finger tapping_relevant stop $(p s>0.901)$, vocalization_relevant stop $(p s>0.067)$, finger tapping_irrelevant stop $(p s>0.907)$ and vocalization_irrelevant stop $(p s>0.999)$. There were significant differences between the go types across stop block and response modality. Specifically, every go type in the relevant stop block had more positive asynchronies compared to those in the irrelevant stop block ( $p s<0.008$ ). Moreover, every go type of the vocalization had more positive asynchronies relative to those go types in the finger tapping $(p s<0.003)$. All pairwise comparisons of this three-way interaction are shown in Appendix E, Tables A4 and A5. 


\section{$2 \times 2 \times 2$ repeated-measures $A N O V A$}

510 After excluding the $1^{\text {st }}$ go after the stop signals, we carried out a smaller repeated measures $2 \times 2$

$511 \times 2$ ANOVA to measure the go asynchronies. This ANOVA contained the within subject factors

512 of 2 stop block types (relevant stop, irrelevant stop), 2 response modalities (finger tapping,

513 vocalization) and 2 phases (phase-1, phase-2). The results revealed that both the factors block

514 type and response modality were statistically significant as well as the two-way interaction

515 between block type and response modality $\left(F(1,29)=15.03, p<0.001, \eta^{2} p=0.35\right)$. Because this

516 two-way interaction explains the two significant factors, we explain the interaction only. All

517 other factors and interactions are described in Appendix F, Table A6. It is important to highlight

518 that unlike the previous $3 \times 2 \times 2 \times 2$ ANOVA, the phase factor was not statistically significant

$519\left(F(1,29)=2.30, p=0.141, \eta^{2}{ }_{p}=0.08\right)$. A finding that shows asynchronies were stable across the

520 whole testing session.

521

522 Post hoc Bonferroni corrected comparisons examining the two-way interaction between stop

523 block and response modality showed that the asynchronies in the relevant stop condition were

524 delayed relative to those of the irrelevant stop condition $(p s<0.001)$. Specifically, asynchronies

525 for finger tapping_relevant stop $(M=-70.24, S E=8.62,95 \% C I=[-87.86$ to -52.62$])$ occurred 58.05

526 ms later compared to those asynchronies for finger tapping_irrelevant stop $(M=-128.29, S E=9.19$,

$52795 \% C I=[-147.07$ to -109.51$])$. Likewise, the asynchronies of vocalization_relevant stop $(M=$ -

$52835.37, S E=7.16,95 \% C I=[-50.01$ to -20.74$])$ occurred $26.10 \mathrm{~ms}$ later compared to the

529 asynchronies of vocalization_irrelevant stop $(M=-61.47, S E=5.92,95 \% C I=[-73.57$ to -49.37$])$.

530 This interaction also showed that the asynchronies of vocalization were significantly different to

531 those of the finger tapping $(p s<0.001)$. Specifically, asynchronies for vocalization _irrelevant stop

532 occurred $66.82 \mathrm{~ms}$ later compared to those asynchronies for finger tapping_irrelevant stop. Similarly,

533 the asynchronies for vocalization_relevant stop occurred $34.87 \mathrm{~ms}$ later in comparison to the

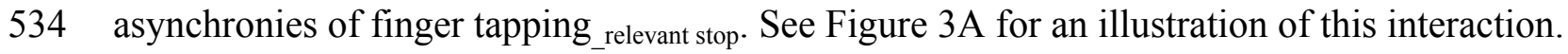



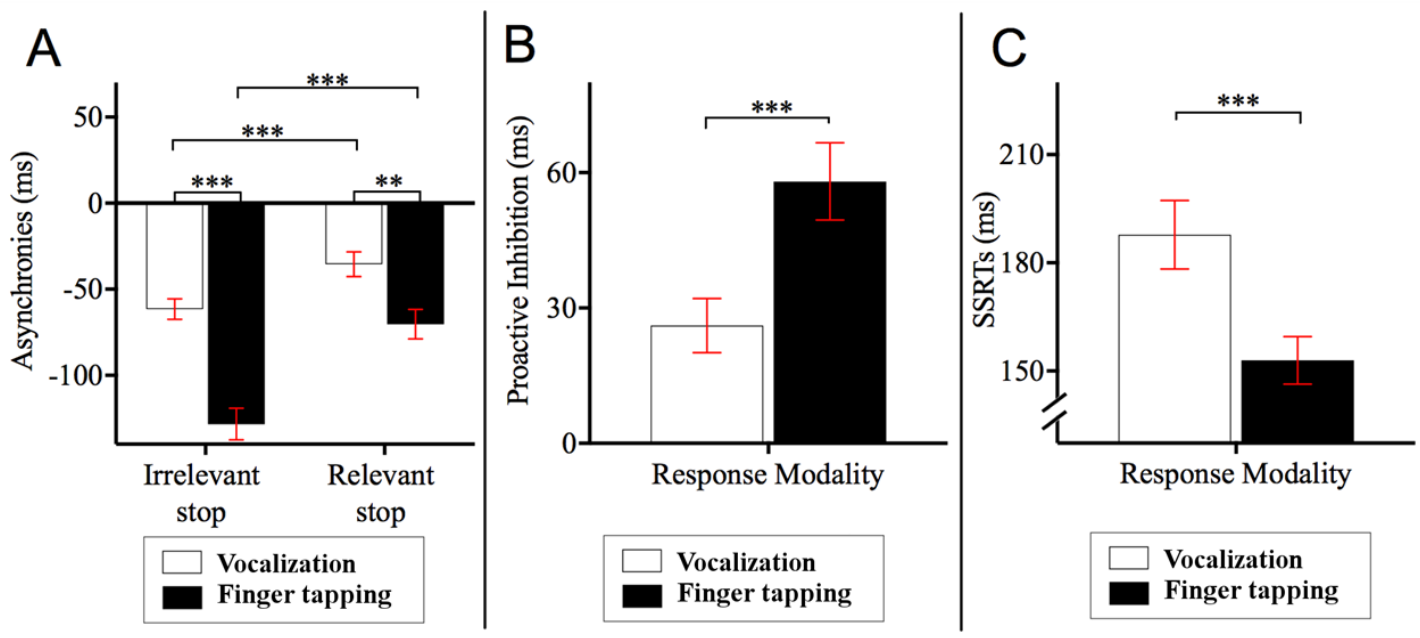

Figure 3. Asynchronies, proactive inhibition and stop signal reaction time (SSRT). A) Two-way interaction between stop block type and response modality from the $2 \times 2 \times 2$ ANOVA on the dependent variable asynchrony. Horizontal dashed line indicates onset of auditory pacing signal.

Stop signals in the relevant stop condition reduced the NMA such that vocalization and finger tapping were delayed. Specifically, for finger

tapping, the stop signals delayed the synchronization by $58.05 \mathrm{~ms}$ whereas for vocalizations, stop signals in the relevant stop condition delayed

the NMA by $26.10 \mathrm{~ms}$. B) Proactive inhibition across response modalities. Proactive inhibition was estimated by subtracting the asynchrony

times in the relevant stop condition from those in irrelevant stop condition. The data showed that stop signals shifted the asynchronies to a lesser

degree for vocalization $(26.11 \mathrm{~ms})$ compared to finger tapping $(58.06 \mathrm{~ms})$. C) SSRTs across response modalities. There were statistically

\subsubsection{Effect of stop-signals on the asynchronies: proactive inhibition}

548 We conducted a paired sample t-test across response modalities for proactive inhibition. This

was calculated as the time difference between relevant and irrelevant stop conditions. These time differences were obtained from the $2^{\text {nd }}$ go after stop signals and all other go after the stop signals. This analysis allowed us to measure the degree that stop signals affected synchronization (i.e., results showed that stop signals during vocalization delayed NMA to a lesser degree $(M=26.11$, $S E=5.99,95 \% C I=[14.64-37.80], p<0.001)$ compared to finger tapping $(M=58.06, S E=$ $8.57,95 \% C I=[40.89-74.62])$. In other words, stop signals did not delay vocalizations as much as they did for finger taps. This result is depicted in Figure 3B. For an analysis of proactive inhibition taking all the go types including the $1^{\text {st }}$ go after stop signals, $2^{\text {nd }}$ go after stop signals 
558 and all other go after the stop signals see Appendix F, Table A7. The analysis shows that the

559 insertion or extraction of the $1^{\text {st }}$ go after the stop signals did not alter the observation that

560 vocalizations induced less proactive inhibition relative to finger tapping.

561

562

\subsubsection{Reactive inhibition - stop signal reaction times (SSRTs)}

563

Reactive inhibition was indexed by the SSRT. SSRT can only be estimated for the relevant stop condition. A paired sample t-test showed that the SSRTs were statistically different between vocalization and finger tapping $(p<0.001)$. This difference showed that finger tapping had significantly shorter SSRT $(M=152.95, S E=6.60,95 \% C I=[140.46-165.60]$ relative to the SSRT of vocalizations $(M=187.81, S E=9.52,95 \% C I=[170.74-205.56])$. An illustration of this analysis is depicted in Figure 3C.

569

570

\subsubsection{Descriptive statistics}

571

A table of the descriptive statistics of go asynchronies, stop signal delay, stop signal reaction

572 time and go misses can be found in Appendix G, Table A8.

573

574

575

1.4.5. Checking assumption of the horse race model

For completeness of this study, we checked the assumption of the horse race model that says that in a failed stop trial, failed stop RT are shorter relative to the go RT. A finding that indicates the go process won the race against the stop process (Logan \& Cowan, 1984). To see this analysis, please go to Appendix $\mathrm{H}$.

579

580

1.5. Discussion

582

The current study investigated a classic finding that occurs in finger tapping to an auditory pacing signal: that taps occur prior to the auditory pacing signal (Dunlap, 1910; Johnson, 1899; Wallin, 1904). An explanation for this phenomenon comes from the Paillard-Fraisse hypothesis (Aschersleben \& Prinz, 1995) and the sensory modal integration theory (Müller et al., 2008) that both conclude that finger tapping to an auditory pacing signal requires two sensory modalities: the tactile/kinaesthetic and auditory. Because the sensory transduction and afferent conduction times vary between these two sensory modalities, there is an inherent offset in the arrival times 
589 afferent signal from the pacing signal and the reafferent signal caused by responding are elicited

590 within the same sensory modality, would the NMA be reduced? This study compared both

591 vocalization and finger tapping to an auditory pacing signal. Because sensory integration would

592 occur in only the auditory modality, it was hypothesized that the NMA would be less for

593 vocalization relative to finger tapping. Secondly, it was hypothesized that stop signals would

594 reduce asynchronies, but to a lesser degree in the vocalization compared to the finger tapping.

595 Our data support both of our hypotheses, these are discussed next.

596

597 The first hypothesis stated that in a control condition where synchronization was important while

598 stopping was irrelevant, the negative asynchrony is smaller for vocalization to an auditory pacing

599 signal compared to the finger tapping to an auditory pacing signal. To test this hypothesis, the

600 control condition had stop signals that were irrelevant. This control condition was called the

601 irrelevant stop condition. We found that while the NMA of the finger tapping occurred at -

$602128.29 \mathrm{~ms}$, those of the vocalizations occurred at $-61.47 \mathrm{~ms}$. In other words, finger tapping had

$60366.82 \mathrm{~ms}$ more negative asynchrony compared to vocalizations.

604

605 These results support the behavioural distinctions that result from the differences between intra606 and inter-modal integration described in Müller et al. (2008). The larger NMA in finger tapping

607 can be explained by the requirement to compare event timing across two sensory modalities. The

608 differences in sensory transduction and afferent conduction times across the sensory modalities

609 for the inter-modal integration produce larger NMA. On the other hand, the shorter NMA for

610 vocalizations is explained by the requirement to compare sensory information within one sensory

611 modality. Müller et al. (2008) found that both finger and toe tapping to a tactile pacing signal

612 (thus an intra-modal integration) had NMA of less than $8 \mathrm{~ms}$ but these differences were not

613 significantly different from the onset of the tactile pacing signal. Whereas both finger and toe

614 tapping to an auditory pacing signal (thus, an inter-modal integration) produced negative

615 asynchronies of about $-40 \mathrm{~ms}$ that were significantly negative relative to the onset of the auditory

616 pacing signal.

617

618 Our second hypothesis, following on from Fischer et al. (2016) stated that the stop signals in the

619 relevant stop blocks reduce the NMA, but this reduction occurs to a lesser degree for 
620 vocalization relative to finger tapping. We found that asynchronies were reduced in the relevant

621 stop blocks compared to the irrelevant stop block in both the vocalization as well as in the finger

622 tapping. Specifically, from the irrelevant to the relevant stop, asynchronies of the finger tapping

623 decreased from $-128.29 \mathrm{~ms}$ to $-70.24 \mathrm{~ms}$ whereas asynchronies of the vocalization decreased

624 from $-61.47 \mathrm{~ms}$ to $-35.37 \mathrm{~ms}$. These results show that stop signals induced proactive inhibition,

625 thereby delaying the onset of the synchronization response and reducing the NMA.

626

627 The next part of this hypothesis was to investigate whether stop signals reduced the NMA to a 628 lesser degree for the intra-modal integration case (i.e., vocalizations to auditory pacing signal)

629 relative to the inter-modal integration case (i.e., finger tapping to auditory pacing signal). The

630 analysis of proactive inhibition showed that for vocalizations, responses were slowed (proactive

631 inhibition) by $26.10 \mathrm{~ms}$ (vocalizations_relevant stop - vocalizations_irrelevant stop) compared to $58.05 \mathrm{~ms}$

632 for finger tapping (finger tapping_relevant stop - finger tapping_irrelevant stop). This finding suggests that

633 vocalization was less perturbed by the stop signals compared to finger tapping. This supports the 634 contention that sensory integration that occurs between modalities (i.e., inter-modal integration)

635 contains a degree of 'play', such that a broader range of tap timings might be tolerated compared 636 to sensory integration within the same sensory modality (i.e., intra-modal integration. Grondin \& 637 Rousseau, 1991; Grondin et al., 2005).

638

639 Given that we found that the NMA for the intra-modal integration was less affected by stop 640 signals compared to inter-modal integration, further studies should assess whether this stability 641 can also be extended to performance variability. In other words, whether intra-modal integration 642 has less tap variability than inter-modal integration. Research suggests that SMS is controlled 643 independently by two error correction processes (phase correction and period correction). Phase 644 correction is considered an automatic process that does not interfere with the tempo of tapping 645 whereas period correction is usually intentional and changes the tempo (see Repp, 2005; Repp \& $646 \mathrm{Su}, 2013$ for reviews). This study also opens up research on delayed feedback in which temporal 647 recalibration is observed when taps become more negative (Cunningham et al., 2001; Heron et 648 al., 2009; Stetson et al., 2006; Sugano et al., 2012). 
650 An interesting aspect to highlight here is that stop signals in the relevant stop blocks made finger 651 tapping and vocalizations more synchronous. This finding shows that stop signals, by inducing 652 proactive inhibition, decreased the NMA of finger tapping and vocalizations - this increment in 653 proactive inhibition improved synchronization. Evidence showing that proactive inhibition plays 654 a crucial role in more accurate synchronizations comes from studies that use explicit

655 performance feedback during SMS. In these experiments, when participants are informed about 656 the size and the direction of the asynchrony (knowledge of results), they are able to tap in exact 657 physical synchrony. However, participants in such experiments report that they have to delay 658 their response in order to perform with greater accuracy (see Aschersleben, 2002 , pp. 67-68). 659 Unlike musically untrained people, trained musicians show significantly less NMA $(\sim-14 \mathrm{~ms})$ 660 suggesting that musical training may require learning to delay the response so it can occur in 661 time (Aschersleben, 2002; Repp, 2004). The link that connects such observations and the current 662 study is that the control of response releases in SMS tasks is likely due to the application of 663 inhibitory control.

664

665 The idea that inhibitory control and rhythmic timing are linked is not a new one. A number of 666 studies note the association between rhythmic ability and executive control dysfunctions, or 667 stages of executive control development (for a review see Repp \& Su, 2013; Rubia et al., 1999). 668 Furthermore, there is a significant overlap in the neural substrates that support inhibitory control 669 and the production of rhythmic tapping tasks (Aron, Robbins, \& Poldrack, 2004; Aron, Robbins, 670 \& Poldrack, 2014; Buhusi \& Meck, 2005; Doumas, Praamstra, \& Wing, 2005; Gross et al., 2002; 671 Jäncke, Loose, Lutz, Specht, \& Shah, 2000; Koch, Oliveri, \& Caltagirone, 2009; Lewis \& Miall, 672 2003; Middleton \& Strick, 2000; O'Boyle, 1999; Wiener, Turkeltaub, \& Coslett, 2010; Witt \& 673 Stevens, 2013). More directly addressing this observation, Witt and Stevens (2013) show that top 674 down influences onto motor cortex from dorsal and ventral prefrontal cortices - particularly in 675 the right hemisphere (Wiener et al., 2010), are associated with the performance of rhythmic 676 tapping. These same areas of the right hemisphere are also strongly associated with inhibitory 677 control (see e.g., Aron et al., 2004; Aron et al., 2014). Moreover, it has previously been 678 demonstrated that longer intervals SMS is related to higher activation in the right dorsolateral 679 pre-frontal cortex (Koch et al., 2009). A transcranial magnetic study found that cortical inhibition 
680 to the motor and premotor cortex reduced the NMA (Doumas et al., 2005). Such studies support 681 our contention that inhibitory control is positively related to accuracy of SMS.

682

683 An incidental finding worth highlighting in this study is that SSRTs were significantly longer for 684 vocalizations relative to finger tapping. This is a finding in line with previous studies that have 685 found vocal responses in the stop signal task exhibit longer SSRTs compared to finger responses 686 (Castro-Meneses et al., 2015), or that there is a trend towards this being the case (Castro687 Meneses et al., 2016; Wessel \& Aron, 2014). Castro-Meneses et al. (2016) presented evidence to 688 suggest that the vocalization system may have weaker reactive inhibition (index by the SSRTs) 689 compared to that of the finger system, which they attributed to inhibitory control differences at the motoneuronal level (Sowman, Flavel, McShane, Miles, \& Nordstrom, 2008).

691

692 One of the limitations of this study is that we assumed that the vocalizations to the auditory 693 pacing signal occurred within the same auditory modality (thus, a case of intra-modal integration). However, the vocalization response can also have tactile/kinaesthetic sensory information when the muscles of the mouth move. We acknowledge this and believe that this could actually explain why the mean negative asynchrony between the vocalization and the auditory pacing signal are not smaller as in Müller et al. (2008). In our present study, vocalizations had a NMA of $61.47 \mathrm{~ms}$ in the irrelevant stop condition, whereas in the intra-modal integration task of Müller et al. (2008) between finger and toe tapping to a tactile pacing signal had a mean negative asynchrony of approximately $8 \mathrm{~ms}$. It could be that the asynchronies of the vocalizations are the average of two sensory modalities: the auditory modality and the

702 tactile/kinaesthetic modality. This idea then would support the sensory accumulator hypothesis (Aschersleben, 2002) that suggests the NMA is a result of a central accumulator of different sensory channels.

705

In support to the sensory accumulator hypothesis, Aschersleben and Prinz (1995) showed that finger and foot tapping to an auditory pacing signal with auditory feedback (i.e., auditory feedback was presented in time with a tap) reduced the negative asynchrony of both finger and foot tapping significantly more compared to the same tasks without auditory feedback. The authors explained this reduction of NMA via the joint-event hypothesis, in which they said that, 
711 because the taps with auditory feedback carried two channels of sensory information:

712 tactile/kinaesthetic and auditory, the brain averages the conduction and sensory transduction

713 times of these two sensory modalities and that is why the NMA reduced but did not disappear

714 completely.

715

716 In the case of our finger tapping to auditory pacing signal, the action of pressing the key button

717 could have also elicited a sound, which could have provided auditory feedback within the same

718 sensory modality as the auditory pacing signal. However, this auditory feedback was reduced

719 because we asked participants to press the key lightly and they also wore ambient noise

720 attenuating headphones (see methods section).

721

722 We also want to emphasize that while we have taken significant steps to ensure adequate

723 accuracy of the reported timing data across modalities (see Appendix A) a level of timing

724 uncertainty remains inherent in our data. Future studies along these lines would be best served by

725 recording the outputs (auditory beats) in parallel with the inputs (microphone and button press)

726 using synchronized DAQ channels in a dedicated recording device. In this way absolute veridical

727 timing relationships can be obtained between inputs and outputs (Schultz \& van Vugt, 2016).

728 Where possible button presses should also be recorded with integrated force transducers in series

729 so that button press timing is not a function of computer port polling. Such methods circumvent

730 the need for complex calibration procedures and ensure the optimal timing accuracy is obtained.

731 Timing accuracy should be considered a limitation of our findings that vocalization exhibits less

732 NMA than finger tapping. However, the interaction between modality (vocal vs. manual) and

733 stop condition (relevant vs. irrelevant) is unaffected by this limitation.

734

735 In conclusion, the current study investigated negative asynchronies of finger tapping and

736 vocalizations to an auditory pacing signal in a modified stop signal task context. When stopping

737 was not required, the data show that vocalization were produced with smaller NMA compared to

738 finger tapping. When stopping was required, proactive inhibition induced a delay in the

739 responses, thereby reducing the NMA. Importantly, this decrement was smaller for vocalizations

740 than for finger tapping. Our data support the Paillard-Fraisse hypothesis, which predicts that

741 negative asynchrony should be smaller for vocalization because this is achieved via an intra- 
742 modal integration. We extend this hypothesis by contending that intra-modal integration should

743 be more sensitive to synchronization discrepancies and therefore would be more resistant to

744 perturbation. Our data show that stop signals did not perturb the synchronization response of

745 vocalization as much they did for finger tapping.

746

747 Acknowledgments

748 We thank Prabhanjali Peters and Jessica Crampton for helping out in data collection. We also

749 thank Jordan Wehrman for his valuable feedback on first drafts of this manuscript. 
750

751

752

753

754

755

756

757

758

759

760

761

762

763

764

765

766

767

768

769

770

771

772

773

774

775

776

777

778

\subsection{References}

Aron, A. R. (2011). From reactive to proactive and selective control: Developing a richer model for stopping inappropriate responses. Biol Psychiatr, 69(12), e55-e68. doi: 10.1016/j.biopsych.2010.07.024

Aron, A. R., Robbins, T. W., \& Poldrack, R. A. (2004). Inhibition and the right inferior frontal cortex. Trends in Cog Sci, 8(4), 170-177. doi: 10.1016/j.tics.2004.02.010

Aron, A. R., Robbins, T. W., \& Poldrack, R. A. (2014). Inhibition and the right inferior frontal cortex: one decade on. Trends in Cog Sci, 18(4), 177-185. doi: 10.1016/j.tics.2013.12.003

Aschersleben, G. (2002). Temporal control of movements in sensorimotor synchronization. Brain Cogn, 48(1), 66-79. doi: 10.1006/brcg.2001.1304

Aschersleben, G., \& Prinz, W. (1995). Synchronizing actions with events: The role of sensory information. Percept \& Psychophys, 57(3), 305-317. doi: 10.3758/BF03213056

Audacity Team. (Copyright (c) 2018). Audacity (R): Free Audio Editor and Recorder [Computer Program] (Version version 1.34-beta ). Retrieved from https://sourceforge.net/projects/audacity/

Billon, M., Bard, C., Fleury, M., Blouin, J., \& Teasdale, N. (1996). Simultaneity of two effectors in synchronization with a periodic external signal. Human Movement Science, 15(1), 2538. doi: 10.1016/0167-9457(95)00037-2

Bolton, T. L. (1894). Rhythm. The american journal of psychology, 6(2), 145-238. doi: $10.2307 / 1410948$

Buhusi, C. V., \& Meck, W. H. (2005). What makes us tick? Functional and neural mechanisms of interval timing. Nat Rev Neurosci, 6, 755. doi: 10.1038/nrn1764

Carroll, C. A., O’Donnell, B. F., Shekhar, A., \& Hetrick, W. P. (2009). Timing dysfunctions in schizophrenia as measured by a repetitive finger tapping task. Brain Cogn, 71(3), 345353. doi: 10.1016/j.bandc.2009.06.009

Castro-Meneses, L. J., Johnson, B. W., \& Sowman, P. F. (2015). The effects of impulsivity and proactive inhibition on reactive inhibition and the go process: insights from vocal and manual stop signal tasks. Front Hum Neurosc, 9. doi: 10.3389/fnhum.2015.00529 
779 Castro-Meneses, L. J., Johnson, B. W., \& Sowman, P. F. (2016). Vocal response inhibition is

780

781

782

783

784

785

786

787

788

789

790

791

792

793

794

795

796

797

798

799

800

801

802

803

804

805

806

807

808 enhanced by anodal tDCS over the right prefrontal cortex. Exp Brain Res. doi: $10.1007 / \mathrm{s} 00221-015-4452-0$

Chen, Y., Ding, M., \& Scott Kelso, J. A. (2001). Origins of Timing Errors in Human Sensorimotor Coordination. Journal of Motor Behavior, 33(1), 3-8. doi: $10.1080 / 00222890109601897$

Chikazoe, J., Jimura, K., Hirose, S., Yamashita, K.-i., Miyashita, Y., \& Konishi, S. (2009). Preparation to inhibit a response complements response inhibition during performance of a stop-signal task. J Neurosci, 29(50), 15870-15877. doi: 10.1523/jneurosci.364509.2009

Cunningham, D. W., Billock, V. A., \& Tsou, B. H. (2001). Sensorimotor Adaptation to Violations of Temporal Contiguity. Psychol Sci, 12(6), 532-535. doi: 10.1111/14679280.d01-17

Doumas, M., Praamstra, P., \& Wing, A. M. (2005). Low frequency rTMS effects on sensorimotor synchronization. Exp Brain Res, 167(2), 238-245. doi: 10.1007/s00221005-0029-7

Dunlap, K. (1910). Reaction to rhythmic stimuli with attempt to synchronize. Psychol Rev, 17(6), 399-416. doi: 10.1037/h0074736

Duque, J., Greenhouse, I., Labruna, L., \& Ivry, R. B. (2017). Physiological Markers of Motor Inhibition during Human Behavior. Trends in Neurosciences, 40(4), 219-236. doi: 10.1016/j.tins.2017.02.006

Etchell, A. C., Sowman, P. F., \& Johnson, B. W. (2012). "Shut up!" An electrophysiological study investigating the neural correlates of vocal inhibition. Neuropsychologia, 50(1), 129-138. doi: 10.1016/j.neuropsychologia.2011.11.009

Falk, S., Müller, T., \& Dalla Bella, S. (2015). Non-verbal sensorimotor timing deficits in children and adolescents who stutter. Front Psychol, 6(847). doi: 10.3389/fpsyg.2015.00847

Fischer, P., Tan, H., Pogosyan, A., \& Brown, P. (2016). High post - movement parietal low - beta power during rhythmic tapping facilitates performance in a stop task. Eur $J$ Neurosci, 44(5), 2202-2213. 
809 Grondin, S., \& Rousseau, R. (1991). Judging the relative duration of multimodal short empty 810 time intervals. Percept \& Psychophys, 49(3), 245-256. doi: 10.3758/bf03214309

811 Grondin, S., Roussel, M.-E., Gamache, P.-L., Roy, M., \& Ouellet, B. (2005). The Structure of

812 Sensory Events and the Accuracy of Time Judgments. Perception, 34(1), 45-58. doi:

$813 \quad 10.1068 / \mathrm{p} 5369$

814 Gross, J., Timmermann, L., Kujala, J., Dirks, M., Schmitz, F., Salmelin, R., \& Schnitzler, A.

815 (2002). The neural basis of intermittent motor control in humans. Proceedings of the

$816 \quad$ National Academy of Sciences, 99(4), 2299-2302. doi: 10.1073/pnas.032682099

817 Heron, J., Hanson, J. V. M., \& Whitaker, D. (2009). Effect before Cause: Supramodal

818 Recalibration of Sensorimotor Timing. PLOS ONE, 4(11), e7681. doi:

$819 \quad 10.1371$ journal.pone.0007681

820 Hove, M. J., Fairhurst, M. T., Kotz, S. A., \& Keller, P. E. (2013). Synchronizing with auditory

821 and visual rhythms: An fMRI assessment of modality differences and modality

822 appropriateness. NeuroImage, 67, 313-321. doi: j.neuroimage.2012.11.032

823 Iversen, J. R., \& Balasubramaniam, R. (2016). Synchronization and temporal processing.

824 Current Opinion in Behavioral Sciences, 8(Supplement C), 175-180. doi:

$825 \quad 10.1016 /$ j.cobeha.2016.02.027

826 Jaffard, M., Longcamp, M., Velay, J.-L., Anton, J.-L., Roth, M., Nazarian, B., \& Boulinguez, P.

827 (2008). Proactive inhibitory control of movement assessed by event-related fMRI.

828 Neurolmage, 42(3), 1196-1206. doi: 10.1016/j.neuroimage.2008.05.041

829 Jäncke, L., Loose, R., Lutz, K., Specht, K., \& Shah, N. J. (2000). Cortical activations during

830 paced finger-tapping applying visual and auditory pacing stimuli. Cognitive Brain

831 Research, 10(1), 51-66. doi: 10.1016/S0926-6410(00)00022-7

832 Johnson, W. S. (1899). Researches in Practice and Habit. Science, 10(250), 527-529.

833 Koch, G., Oliveri, M., \& Caltagirone, C. (2009). Neural networks engaged in milliseconds and

834 seconds time processing: evidence from transcranial magnetic stimulation and patients

835 with cortical or subcortical dysfunction. Philosophical Transactions of the Royal Society

836 B: Biological Sciences, 364(1525), 1907-1918. doi: 10.1098/rstb.2009.0018

837 Large, E. W., \& Jones, M. R. (1999). The dynamics of attending: How people track time-varying $838 \quad$ events. Psychol Rev, 106(1), 119-159. doi: 10.1037/0033-295X.106.1.119 
839 Lewis, P. A., \& Miall, R. C. (2003). Distinct systems for automatic and cognitively controlled

840

841

842

843

844

845

846

847

848

849

850

851

852

853

854

855

856

857

858

859

860

861

862

863

864

865

866

867

868 time measurement: evidence from neuroimaging. Current Opinion in Neurobiology, 13(2), 250-255. doi: 10.1016/S0959-4388(03)00036-9

Logan, G. D. (1994). On the ability to inhibit thought and action: A users' guide to the stop signal paradigm. In D. Dagenbach \& T. H. Carr (Eds.), Inhibitory processes in attention, memory, and language (pp. 189-239). San Diego, CA, US: Academic Press.

Logan, G. D., \& Cowan, W. B. (1984). On the ability to inhibit thought and action: A theory of an act of control. Psychol Rev, 91(3), 295-327. doi: 10.1037/0033-295x.91.3.295

Logan, G. D., Schachar, R. J., \& Tannock, R. (1997). Impulsivity and inhibitory control. Psychol Sci, 8(1), 60-64. doi: 10.1111/j.1467-9280.1997.tb00545.x

London, J. (2002). Cognitive Constraints on Metric Systems: Some Observations and Hypotheses. Music Perception: An Interdisciplinary Journal, 19(4), 529-550. doi: 10.1525/mp.2002.19.4.529

Lumpkin, E. A., \& Caterina, M. J. (2007). Mechanisms of sensory transduction in the skin. Nature, 445(7130), 858-865. doi: 10.1038/nature05662

Maes, P.-J., Wanderley, M. M., \& Palmer, C. (2015). The role of working memory in the temporal control of discrete and continuous movements. Exp Brain Res, 233(1), 263-273. doi: 10.1007/s00221-014-4108-5

Middleton, F. A., \& Strick, P. L. (2000). Basal ganglia and cerebellar loops: motor and cognitive circuits. Brain Research Reviews, 31(2), 236-250. doi: 10.1016/S0165-0173(99)00040-5

Müller, K., Aschersleben, G., Schmitz, F., Schnitzler, A., Freund, H.-J., \& Prinz, W. (2008). Inter- versus intramodal integration in sensorimotor synchronization: a combined behavioral and magnetoencephalographic study. Exp Brain Res, 185(2), 309-318. doi: 10.1007/s00221-007-1155-1

O'Boyle, D. J. (1999). What is the source of the mean anticipatory synchronisation error during temporal tracking?: Commentary on Müller et al. In T. B. Gisa Aschersleben \& M. Jochen (Eds.), Advances in Psychology (Vol. Volume 129, pp. 259-263): North-Holland.

Osman, A., Kornblum, S., \& Meyer, D. E. (1986). The point of no return in choice reaction time: Controlled and ballistic stages of response preparation. J Exp Psychol-Hum Percep Perform, 12(3), 243-258. doi: 10.1037/0096-1523.12.3.243 
869 Osman, A., Kornblum, S., \& Meyer, D. E. (1990). Does motor programming necessitate

870

871

872

873

874

875

876

877

878

879

880

881

882

883

884

885

886

887

888

889

890

891

892

893

894

895

896

897

898 response execution? J Exp Psychol -Hum Percep Perform, 16(1), 183-198. doi: 10.1037/0096-1523.16.1.183

Pikovsky, A., Rosenblum, M., \& Kurths, J. (2003). Synchronization: a universal concept in nonlinear sciences (Vol. 12): Cambridge university press.

Repp, B. H. (2004). On the nature of phase attraction in sensorimotor synchronization with interleaved auditory sequences. Human Movement Science, 23(3-4), 389-413. doi: 10.1016/j.humov.2004.08.014

Repp, B. H. (2005). Sensorimotor synchronization: A review of the tapping literature. Psychon Bull \& Rev, 12(6), 969-992. doi: 10.3758/BF03206433

Repp, B. H., \& Su, Y.-H. (2013). Sensorimotor synchronization: A review of the tapping literature. Psychon Bull \& Rev, 12(6), 969-992. doi: 10.3758/bf03206433

Rubia, K., Taylor, A., Taylor, E., \& Sergeant, J. A. (1999). Synchronization, anticipation, and consistency in motor timing of children with dimensionality defined attention deficit hyperactivity behaviour. Perceptual and motor skills, 89(3f), 1237-1258.

Schulze, H.-H., \& Vorberg, D. (2002). Linear Phase Correction Models for Synchronization: Parameter Identification and Estimation of Parameters. Brain Cogn, 48(1), 80-97. doi: 10.1006/brcg.2001.1305

Sowman, P. F., Flavel, S. C., McShane, C. L., Miles, T. S., \& Nordstrom, M. A. (2008). Transcranial magnetic stimulation reduces masseter motoneuron pool excitability throughout the cortical silent period. Clin Neurophysiol, 119(5), 1119-1129. doi: 10.1016/j.clinph.2007.12.019

Stetson, C., Cui, X., Montague, P. R., \& Eagleman, D. M. (2006). Motor-Sensory Recalibration Leads to an Illusory Reversal of Action and Sensation. Neuron, 51(5), 651-659. doi: 10.1016/j.neuron.2006.08.006

Stevens, L. T. (1886). On the time-sense. Mind(43), 393-404.

Strogatz. (2003). Synchronization: A universal concept in nonlinear sciences. Physics Today, 56(1), 47. doi: 10.1063/1.1554136

Studenka, B. E., Zelaznik, H. N., \& Balasubramaniam, R. (2012). The distinction between tapping and circle drawing with and without tactile feedback: An examination of the 
899

900

901

902

903

904

905

906

907

908

909

910

911

912

913

914

915

916

917

918

919

920

921

922

923

924

925

926

927

sources of timing variance. The Quarterly Journal of Experimental Psychology, 65(6), 1086-1100. doi: 10.1080/17470218.2011.640404

Sugano, Y., Keetels, M., \& Vroomen, J. (2012). The Build-Up and Transfer of Sensorimotor Temporal Recalibration Measured via a Synchronization Task. Front Psychol, 3, 246. doi: 10.3389/fpsyg.2012.00246

Vaughan, J., Rosenbaum, D. A., Diedrich, F. J., \& Moore, C. M. (1996). Cooperative selection of movements: The optimal selection model. Psychological Research, 58(4), 254-273. doi: $10.1007 / \mathrm{bf00447072}$

Verbruggen, F., Chambers, C. D., \& Logan, G. D. (2013). Fictitious inhibitory differences: how skewness and slowing distort the estimation of stopping latencies. Psychol Sci, 24(3), 352-362. doi: 10.1177/0956797612457390

Verbruggen, F., \& Logan, G. D. (2009a). Models of response inhibition in the stop-signal and stop-change paradigms. Neurosci \& Biobehav Rev, 33(5), 647-661. doi: 10.1016/j.neubiorev.2008.08.014

Verbruggen, F., \& Logan, G. D. (2009b). Proactive adjustments of response strategies in the stop-signal paradigm. J Exp Psychol -Hum Percep Perform, 35(3), 835-854. doi: $10.1037 / \mathrm{a} 0012726$

Wallin, J. E. W. (1904). Researches on Rhythmic Action. Psychol Bull, 1(6), 188-194. doi: $10.1037 / \mathrm{h} 0072068$

Wessel, J. R., \& Aron, A. R. (2014). It's not too late: The onset of the frontocentral P3 indexes successful response inhibition in the stop-signal paradigm. Psychophysiol, 52(4), 472480. doi: 10.1111/psyp.12374

Wiener, M., Turkeltaub, P., \& Coslett, H. B. (2010). The image of time: A voxel-wise metaanalysis. NeuroImage, 49(2), 1728-1740. doi: 10.1016/j.neuroimage.2009.09.064

Wing, A. M. (2002). Voluntary timing and brain function: an information processing approach. Brain Cogn, 48(1), 7-30. doi: 10.1006/brcg.2001.1301

Witt, S. T., \& Stevens, M. C. (2013). The role of top-down control in different phases of a sensorimotor timing task: a DCM study of adults and adolescents. Brain imaging and behavior, 7(3). doi: 10.1007/s11682-013-9224-5 
928

929

930

931

932

933

934 We used PowerLab 8/35 to measure the veridical times of inputs and outputs.

935 (https://www.adinstruments.com/products/powerlab). We conducted tests with a sampling rate of $9361 \mathrm{Khz}$ which would mean a possible error in accuracy of $\sim 1 \mathrm{~ms}$. Testing was conducted under the 937 same lab conditions as the report.
939

940

\section{1}

942

943

944

945

946 947

948 In the first step, we measured timing in the electronic part of the loop. By triggering the scope

949 function in Powerlab we recorded simultaneously both output and input A (as depicted in Figure

950 A2). The microphone of input A recorded the same output (i.e., the beat). We found a timing 951 difference on average of less than $1 \mathrm{~ms}$ (below the sampling accuracy). *We also conducted the

Figure A1. Experimental circuit representing output and inputs relative to stimulus computer. Output refers to the beat sound. It was elicited through headphones via the stimulus computer's soundcard. The inputs represent response modalities. Input A refers to vocalizations. These were picked up by a microphone and sent back to the stimulus computer to be recorded. The input B indicates finger taps. These responses were picked up by a keypad and sent back to the stimulus computer.

\section{Step 1: Time differences between output and input A (vocalizations) recorded in PowerLab}

Input A: Vocalization, sent through microphone

Input B: button press, sent through

Keypad 
952 same procedure with an oscilloscope - the results were the same, and for analysis purposes the 953 output from the Powerlab is easier to deal with*

954

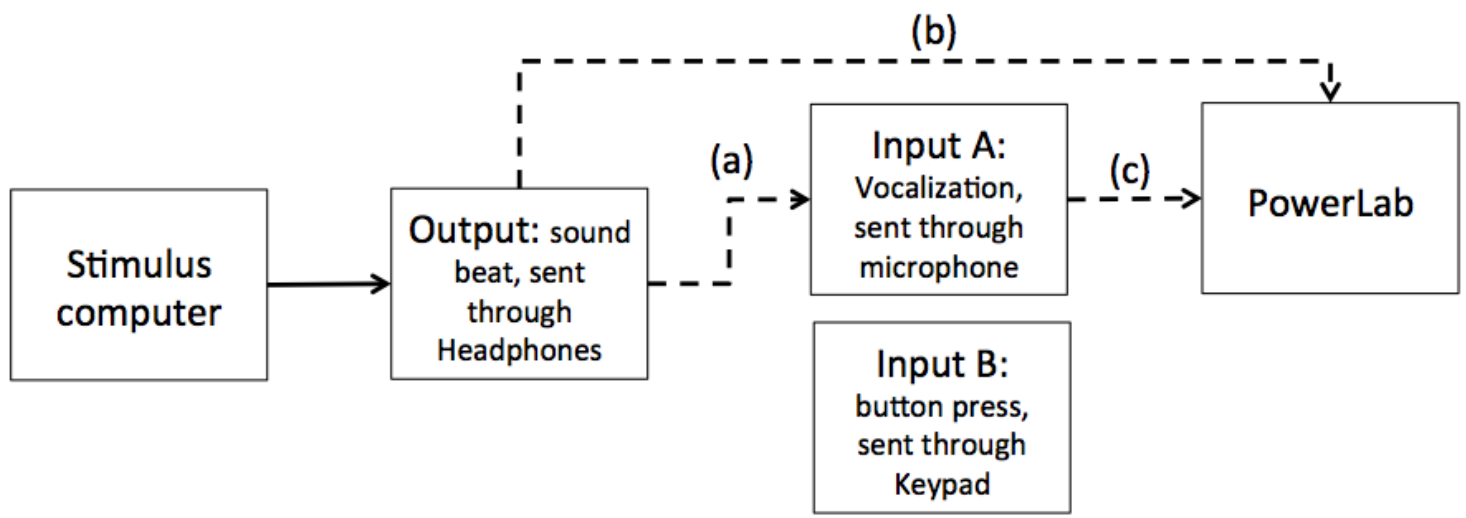

955

956

957

958

959

960

961

962

963

964

965

966

967

968

969

970

971

972 This meant that to adjust the recorded time of the vocalizations in Presentation software to a

973 more veridical time, we needed to subtract $67.43 \mathrm{~ms}$ of the vocalizations.

Figure A2. Output and Input A recorded in PowerLab. The dashed line (a) shows that Input A is recording the same output sound. The dashed lines ( $b$ and $c$ ) show that the output and input A respectively are recorded in PowerLab simultaneously. PowerLab recorded a timing difference of less than $1 \mathrm{~ms}$ between a and $\mathrm{b}$.

\section{Step 2: Time differences between output and input $A$ (vocalizations) recorded in} Presentation software

As depicted in Figure A3, in Presentation software we compared the time differences of the output and input A, when input A was recording the same output (i.e., the beat). We found that from the time the stimulus computer - specifically the experimental software elicited an output, $67.43 \mathrm{~ms}$ later (range between 72.5 and $62.2 \mathrm{~ms}$ ) input A was recorded in the stimulus computer.

We know from step 1 that the time delay between the output and input A in PowerLab is less than $1 \mathrm{~ms}$, so this delay can be attributed to a difference in experimental stimulus time vs. the actual output time from the stimulus computer's soundcard. 


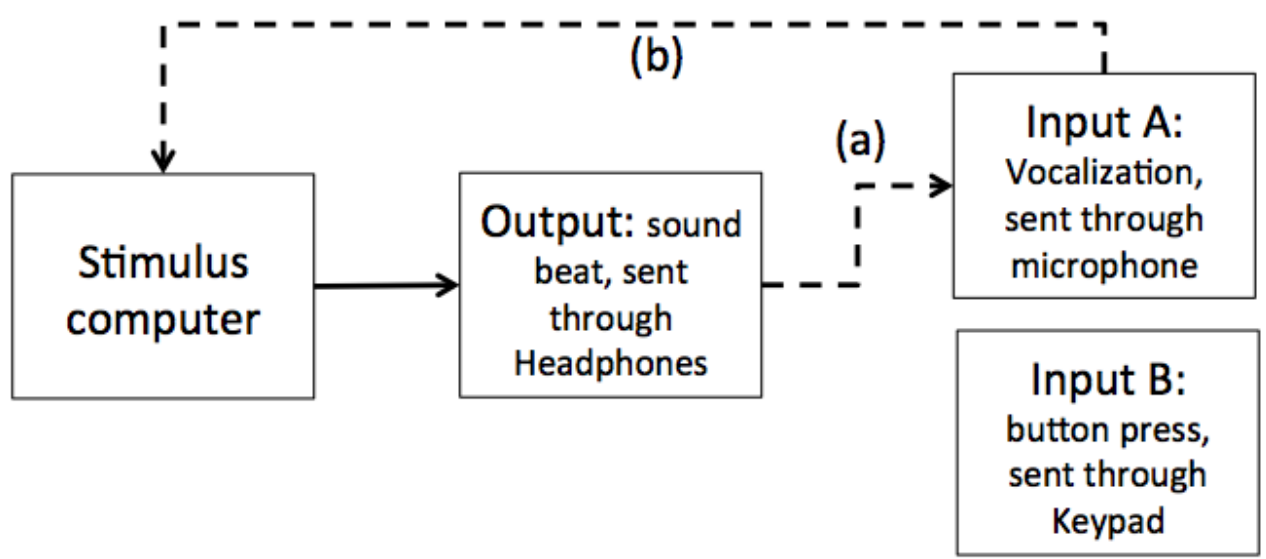

974

975

976

977

978

979

980

981

982

983

984

985

986

987

988

989

990

991

992

993

994

995

996

997

Figure A3. Output and Input A recorded in Presentation Software. The dashed line (a) shows that input A is recording the output sound. The dashed line (b) shows that input A is recorded in stimulus computer.

\section{Step 3: Time differences between input $A$ (vocalizations) and input B (finger taps) recorded} in Presentation software

To test if any significant timing difference existed between input B (button press) and input A (vocalization), the sound of the button press was enhanced by striking the button hard enough to make a recordable response. Then, input B recorded the sound elicited of a button press (see Figure A4 for an illustration of this).

Times recorded for the input B (button press) and the input A (sound) were minimally different (average of $8.4 \mathrm{~ms}$, range between 4.99 and $10.36 \mathrm{~ms}$ ). The recorded time of the button press (input B) was registered in average $8.4 \mathrm{~ms}$ later than the microphone (input A). This delay in input $B$ is very close to the manufacturers latency claim of $\sim 4 \mathrm{~ms}$ for the Cedrus keypad device. The extra time is likely due to the mechanical coupling time, which we assume the manufacturer does not count.

This means that to calibrate input $\mathrm{A}$ and $\mathrm{B}$, we needed to substract $8.4 \mathrm{~ms}$ of the button press (input B) to make it comparable to input A (vocalizations). Moreover, in step 2 we already found that input A (vocalizations) had a delayed time of $67.43 \mathrm{~ms}$. This means that further $67.43 \mathrm{~ms}$ would be need to be subtracted from input B (button press). 
998

999

1000

1001

1002

1003

1004

1005

1006

1007

1008

1009

1010

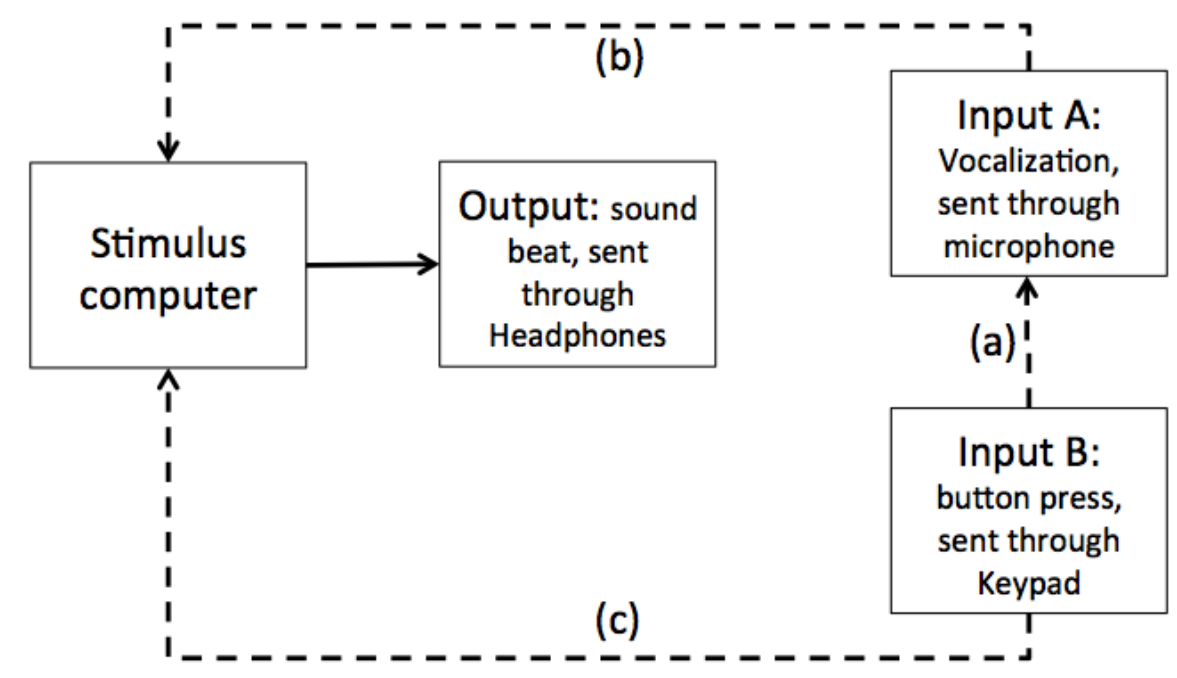

Figure A4. Input A recording Input B in Presentation Software. The dashed line (a) shows that input A is recording the sound emitted from Input B. The dashed lines ( $\mathrm{b}$ and $\mathrm{c}$ ) show that both inputs are recorded in stimulus computer.

\section{Step 4: Time differences between input A (vocalizations) and accelerometer mounted on} input B (button press) recorded in PowerLab

We further calibrated the method of recording Input B (button press) via the input A (microphone picking button press' sound) and via an accelerometer mounted on the button (input $\mathrm{C}$ as shown on Figure A5). We used the accelerometer input to trigger the scope and record the sound of input $\mathrm{A}$ on the second channel. The time difference between the accelerometer and Input A was in the order of $1 \mathrm{~ms}$, indicating that the microphone input was a very good proxy for the mechanical contact time. 


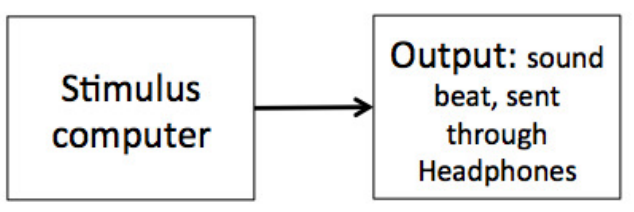

1012

1013

101

101

1016

1017

1018 Conclusion:

1019 To calibrate the two response modalities, we subtracted $8.4 \mathrm{~ms}$ of the button presses to make

1020

1021

1022 $67.43 \mathrm{~ms}$ from both vocalizations and finger taps.

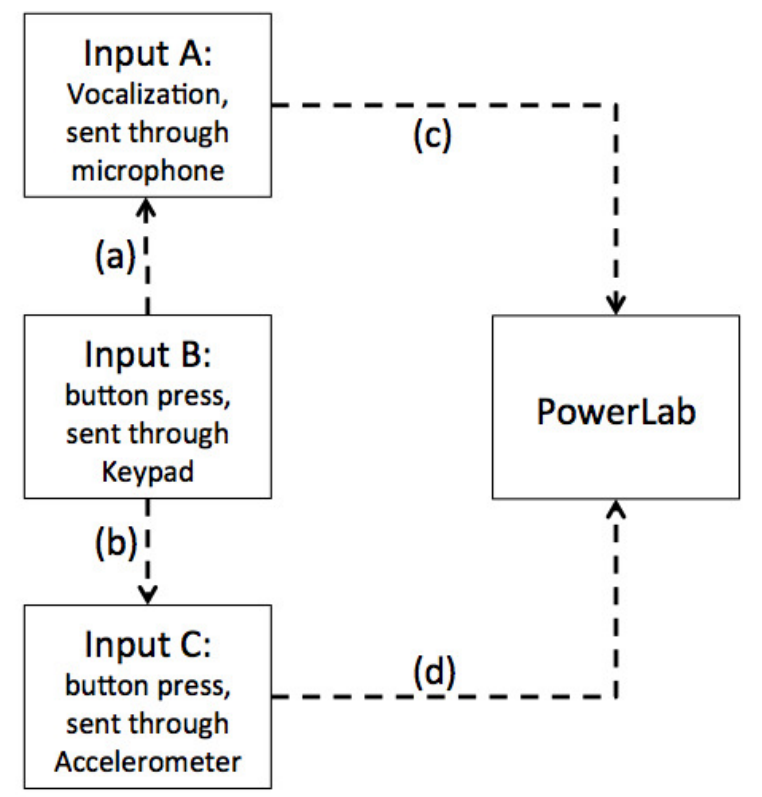

Figure A5. Input B is recorded via both input A and Input C (i.e., accelerometer) in PowerLab. The dashed line (a) shows that input A (i.e., the microphone) is recording the sound emitted from Input B (i.e., the button press). The dashed line (b) indicates that input $\mathrm{C}$, which is the accelerometer, records activity of Input B. The dashed lines (c and d) show input $A$ and Input $C$ are recorded in PowerLab simultaneously. The results show that a button press recorded through an accelerometer and the sound emitted of it had less than 1ms timing difference in PowerLab. them comparable to vocalizations. Moreover, to adjust for the time delay between when a response was given and the time that that response was registered in presentation, we subtracted 


\section{Appendix B. Comparison of the horse race model with the current study's task}

1025

A

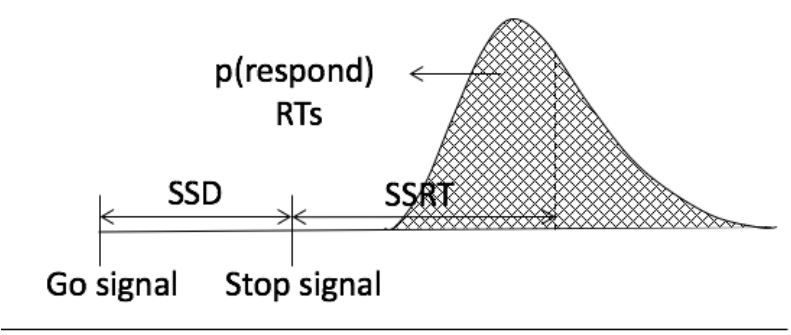

B

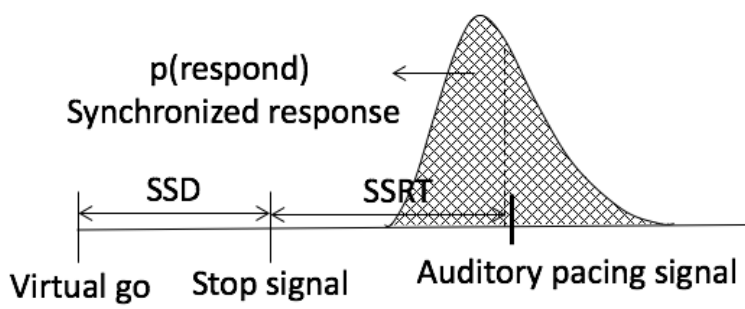

1027

Figure A6. Graphical representation of the independent horse-race model and the current study's task

1028

A) Traditional stop-signal task taken from Logan and Cowan (1984). The graph shows that a go signal triggers a go-

1029 response while a stop signal triggers withholding of the go-response. The responses are a reaction time, which are considered the end of the go-response. The area under the curve to the left of the dashed line represents probability of responding in stop trials [p(respond)] or unsuccessful stopping. B) Variables of the stop signal task combined with a synchronization task. In this task, there is no go signal, but a virtual go signal is estimated that must trigger

1033 the initiation of the synchronized response. It is inserted post hoc to allow calculation of the stop signal reaction time

1034 (SSRT). A stop signal triggers withholding of the go-response. Synchronized responses consisted of a finger taps or 1035 a vocalization. These are considered the end of the go.

1036 
1037 Appendix C. Significant factors and interactions of the $3 \times 2 \times 2 \times 2$ ANOVA

1038

1039 Following up from the results section, here we describe the factors and interactions that were

1040 significant but did not involve the go type factor for the $3 \times 2 \times 2 \times 2$ ANOVA. There were three

1041 significant factors and one interaction: stop block type, response modality, phase and the

1042 interaction between stop block type and response modality. Because the interaction describes

1043 two factors (stop block and response modality), we describe the phase factor and the interaction

1044 only.

1045

1046 The phase factor showed that the asynchronies in phase-1 $(M=-68.64, S E=5.69,95 \% C I=[-$

104780.27 to -57.02$]$ ) occurred closer to the pacing signal compared to those asynchronies of phase-2

$1048(M=-79.53, S E=6.95,95 \% C I=[-93.75$ to -65.32$])$. This phase factor finding is also explained

1049 by the interaction between go type and phases described in the manuscript, in which the longer

1050 asynchronies in phase-1 were driven by the 1st go-trial after stop signals.

1051

1052 The interaction between stop block type and response modality showed that asynchronies from

1053 the relevant stop block occurred later compared to those of the irrelevant stop block across each

1054 response modality as follows: asynchronies of finger tapping relevant stop $(M=-69.23, S E=6.92$,

$105595 \% C I=[-83.36$ to -55.09$])$ occurred later than those from finger tapping irrelevant stop $(M=$ -

$1056129.32, S E=8.95,95 \% C I=[-147.62$ to -111.02$], p<0.001)$. Asynchronies of

1057 vocalization_relevant stop $(M=-36.51, S E=6.89,95 \% C I=[-50.58$ to -22.43$])$ occurred later than

1058 those asynchronies of vocalization_irrelevant stop $(M=-61.30, S E=5.47,95 \% C I=[-72.49$ to -

$105950.11], p<0.001)$. Across response modalities, this interaction showed that asynchronies of

1060 finger tapping were significantly more negative compared to those asynchronies of vocalizations

1061 in the relevant stop blocks $(p<0.001)$ as well as in the irrelevant stop blocks $(p<0.001)$. This

1062 same finding was found in the smaller $2 \times 2 \times$ ANOVA.

1063

1064 To sum up, we carried out this lengthy and complex 3 × 2 × 2 × 2 ANOVA to verify whether stop

1065 signals appearance interfered with the synchronization. We did not want to present data where

1066 participants lost the pacing rhythm because then, this would not represent a SMS effect. The data

1067 from the interaction between go type and phase revealed that the $1^{\text {st }}$ go in phase- 1 was 
1068 significantly different from the $2^{\text {nd }}$ go and all other go across phases 1 and 2 . This was the reason

1069 for which we excluded the 1 st go to carry out a simpler 2 × 2 × 2 ANOVA.

1070

1071 Table A1: $3 \times 2 \times 2 \times 2$ ANOVA of the go asynchronies

\begin{tabular}{llrr}
\hline \multicolumn{1}{c}{ Factor and interaction } & \multicolumn{1}{c}{$\begin{array}{c}\boldsymbol{F} \text { value (degrees of } \\
\text { freedom) }\end{array}$} & $\begin{array}{r}\text { P-value } \\
\text { Pquartial eta- }\end{array}$ \\
\hline 3 go types & $F(1.6,43.7)=0.16$ & 0.793 & .01 \\
2 stop block types & $F(1,29)=79.31$ & $<\mathbf{0 . 0 0 1}$ & .74 \\
2 response-modalities & $F(1,29)=95.80$ & $<\mathbf{0 . 0 0 1}$ & .77 \\
2 phases & $F(1,29)=6.20$ & $\mathbf{0 . 0 1 9}$ & .18 \\
Go type $\times$ stop block type & $F(1.3,36.1)=0.03$ & 0.915 & .01 \\
Go type $\times$ response-modality & $F(1.5,43.5)=0.90$ & 0.390 & .01 \\
Stop block type $\times$ response-modality & $F(1,29)=22.60$ & $<\mathbf{0 . 0 0 1}$ & .44 \\
Go type $\times$ stop block type $\times$ response modality & $F(1.7,48.5)=4.59$ & $\mathbf{0 . 0 2 1}$ & .14 \\
Go type $\times$ phases & $F(2,58)=5.88$ & $\mathbf{0 . 0 0 5}$ & .17 \\
Stop block type $\times$ phases & $F(1,29)=0.05$ & 0.830 & .01 \\
Go type $\times$ stop block type $\times$ phases & $F(2,58)=1.45$ & 0.245 & .05 \\
Response modality $\times$ phases & $F(1,29)=0.74$ & 0.399 & .03 \\
Go type $\times$ response modality $\times$ phases & $F(2,58)=2.98$ & 0.059 & .10 \\
Stop block type $\times$ response modality $\times$ phases & $F(1,29)=0.24$ & 0.629 & .01 \\
Go type $\times$ stop block type $\times$ response modality $\times$ phases & $F(2,58)=0.47$ & 0.631 & .02
\end{tabular}

- Greenhouse-Geisser corrected 
1073 Appendix D. Descriptive statistics and pairwise comparisons of the two-way interaction

1074 between go type and phase of the $3 \times 2 \times 2 \times 2$ ANOVA

1075

1076 Table A2. Descriptive statistics for the two-way interaction between go type and phase of

1077 the $3 \times 2 \times 2 \times 2$ repeated measures ANOVA on the asynchronies

\begin{tabular}{l|c|c|c|c|c|c}
\hline \multirow{2}{*}{ Go type } & \multicolumn{3}{c|}{ Phase 1 } & \multicolumn{3}{c}{ Phase 2 } \\
& Mean & SE & $\mathbf{9 5 \%}$ CI & Mean & SE & 95\% CI \\
\hline $1^{\text {st }}$ go & -65.05 & 5.84 & -76.98 to -53.12 & -82.75 & 6.98 & -97.02 to -68.48 \\
$2^{\text {nd }}$ go & -70.89 & 6.22 & -83.60 to -58.19 & -79.55 & 7.45 & -94.78 to -64.33 \\
All other go & -69.99 & 6.30 & -82.87 to -57.11 & -76.29 & 7.72 & -92.07 to -60.52 \\
\hline
\end{tabular}

$1078 \mathrm{SE}=$ standard error of the mean; $95 \% \mathrm{CI}=95 \%$ confidence intervals

1080 Table A3. Pairwise comparisons for the two-way interaction between go type and phase of

1081 the $3 \times 2 \times 2 \times 2$ repeated measures ANOVA on the asynchronies

\begin{tabular}{lr}
\hline \multicolumn{1}{c}{ Pairwise comparison } & $p$-value \\
\hline 1st go_phase_1 vs. 2nd go_phase_1 & 0.409 \\
1st go_phase_1 vs. All other go_phase_1 & 0.930 \\
2nd go_phase_1 vs. All other go_phase_1 & 0.999 \\
1st go _phase_2 vs. 2nd go _phase_2 & 0.999 \\
1st go_B2 vs. All other go_phase_2 & 0.546 \\
2nd go_B2 vs. All other go_phase_2 & 0.946 \\
1st go_phase_1 vs. 1st go_phase_2 & $<\mathbf{0 . 0 0 1}$ \\
2nd go_phase_1 vs. 2nd go_phase_2 & 0.109 \\
All other go _phase_1 vs. All other go_phase_2 & 0.213 \\
\hline
\end{tabular}

1082 
1083 Appendix E. Descriptive statistics and pairwise comparisons of the three-way interaction between go type, stop block and response modality of the $3 \times 2 \times 2 \times 2$ ANOVA

1085

Table A4. Descriptive statistics of the three-way interaction between go type, stop block and response modality of the $3 \times 2 \times 2 \times 2$ repeated measures ANOVA on the asynchronies

\begin{tabular}{|c|c|c|c|c|c|c|}
\hline \multirow[b]{2}{*}{ Go type } & \multicolumn{3}{|c|}{ Relevant stop } & \multicolumn{3}{|c|}{ Irrelevant stop } \\
\hline & Mean & SE & $95 \% \mathrm{CI}$ & Mean & SE & $95 \% \mathrm{CI}$ \\
\hline $1^{\text {st }}$ go_finger tapping & -69.07 & 7.19 & -83.76 to -54.38 & -129.94 & 9.05 & -148.45 to -111.43 \\
\hline $2^{\text {nd }}$ go $_{\text {finger tapping }}$ & -65.68 & 8.46 & -82.98 to -48.38 & -131.61 & 9.96 & -152.02 to -111.29 \\
\hline All other go_finger tapping & -72.94 & 9.67 & -92.71 to -53.16 & -126.37 & 9.26 & -145.3 to -107.43 \\
\hline $1^{\text {st }}$ go_vocalization & -36.09 & 8.10 & -52.63 to -19.54 & -60.50 & 5.19 & -71.11 to -49.90 \\
\hline $2^{\text {nd }}$ go_vocalization & -41.66 & 7.40 & -56.78 to -26.55 & -61.89 & 5.22 & -72.56 to -51.23 \\
\hline All other go vocalization & -31.77 & 7.40 & -46.89 to 16.65 & -61.50 & 6.38 & -74.53 to -48.46 \\
\hline
\end{tabular}

$1088 \mathrm{SE}=$ standard error of the mean; $95 \% \mathrm{CI}=95 \%$ confidence intervals

Table A5. Pairwise comparisons for the three-way interaction between go type, stop block and response modality of the $3 \times 2 \times 2 \times 2$ repeated measures ANOVA on the asynchronies

\begin{tabular}{|c|c|}
\hline Pairwise comparison & $p$-value \\
\hline \multicolumn{2}{|c|}{ Differences across go types, and within response modality and stop block } \\
\hline $1 \mathrm{st}$ go_finger tapping_relevant stop VS. 2nd go_finger tapping_relevant stop & 0.999 \\
\hline 1 st go_finger tapping_relevant stop VS. All other go_finger tapping_relevant stop & 0.999 \\
\hline 2 nd go_finger tapping_relevant stop VS. All other go_finger tapping_relevant stop & 0.902 \\
\hline 1 st go_vocalization_relevant stop VS. 2 nd go_vocalization_relevant stop & 0.856 \\
\hline 1st go_vocalization_relevant stop VS. All other go_vocalization_relevant stop & 0.999 \\
\hline 2 nd go_vocalization_relevant stop VS. All other go_vocalization_relevant stop & 0.067 \\
\hline 1st go_finger tapping_irrelevant stop Vs. 2nd go_finger tapping_irrelevant stop & 0.999 \\
\hline 1st go_finger tapping_irrelevant stop VS. All other go_finger tapping_irrelevant stop & 0.999 \\
\hline 2nd go _finger tapping_irrelevant stop VS. All other go finger tapping_irrelevant stop & 0.908 \\
\hline 1 st go_vocalization_irrelevant stop VS. 2nd go_vocalization_irrelevant stop & 0.999 \\
\hline 1st go_vocalization_irrelevant stop VS. All other go_vocalization_irrelevant stop & 0.999 \\
\hline 2nd go_vocalization_irrelevant stop VS. All other go_vocalization_irrelevant stop & 0.999 \\
\hline Differences across stop block, and within response modality & \\
\hline
\end{tabular}




\begin{tabular}{|c|c|}
\hline Pairwise comparison & $p$-value \\
\hline 1st go_finger tapping_relevant stop VS. 1st go_finger tapping_irrelevant stop & $<0.001$ \\
\hline 1 st go_vocalization_relevant stop VS. 1st go_vocalization_irrelevant stop & 0.002 \\
\hline 2nd go_finger tapping_relevant stop vs. 2nd go_finger tapping_irrelevant stop & $<0.001$ \\
\hline 2nd go_vocalization_relevant stop Vs. 2 nd go_vocalization_irrelevant stop & 0.007 \\
\hline All other go_finger tapping_relevant stop VS. All other go_finger tapping_irrelevant stop & $<0.001$ \\
\hline All other go_vocalization_relevant stop Vs. All other go_vocalization_irrelevant stop & $<0.001$ \\
\hline \multicolumn{2}{|l|}{ Differences across stop blocks, and within response modality and go type } \\
\hline 1st go_finger tapping_relevant stop VS. 1st go_vocalization_relevant stop & $<0.001$ \\
\hline 1st go_finger tapping_irrelevant stop VS. 1st go_vocalization_irrelevant stop & $<0.001$ \\
\hline 2 nd go_finger tapping_relevant stop Vs. 2nd go_vocalization_relevant stop & 0.002 \\
\hline 2nd go_finger tapping_irrelevant stop Vs. 2nd go_vocalization_irrelevant stop & $<0.001$ \\
\hline All other go _finger tapping_relevant stop VS. All other go _vocalization_relevant stop & $<0.001$ \\
\hline All other go finger tapping_irrelevant stop VS. All other go_vocalization_irrelevant stop & $<0.001$ \\
\hline
\end{tabular}

1092 
1093 Appendix F. Factors and interactions of the 2 x 2 x 2 ANOVA

1094

1095 Table A6: 2 × 2 × 2 ANOVA of the go asynchronies

\begin{tabular}{llrr}
\hline \multicolumn{1}{c}{ Factor and interaction } & \multicolumn{1}{c}{$\begin{array}{c}\boldsymbol{F} \text { value (degrees of } \\
\text { freedom) }\end{array}$} & p-value & $\begin{array}{c}\text { Partial eta- } \\
\text { square }\end{array}$ \\
\hline 2 stop block types & $F(1,29)=46.97$ & $<\mathbf{0 . 0 0 1}$ & .62 \\
2 response-modalities & $F(1,29)=96.98$ & $<\mathbf{0 . 0 0 1}$ & .77 \\
2 phases & $F(1,29)=2.30$ & 0.141 & .08 \\
Stop block type $\times$ response-modality & $F(1,29)=15.03$ & $<\mathbf{0 . 0 0 1}$ & .35 \\
Stop block type $\times$ phases & $F(1,29)=0.14$ & 0.717 & .01 \\
Response modality $\times$ phases & $F(1,29)=0.15$ & 0.707 & .01 \\
Stop block type $\times$ response modality $\times$ phases & $F(1,29)=0.69$ & 0.414 & .03 \\
\hline
\end{tabular}

1096 
1097 Appendix G. Results of paired-sample t-test of proactive inhibition with all go

1098 synchronization distribution

1099

1100 Table A7: Results of paired-sample t-test of proactive inhibition

\begin{tabular}{cc} 
& $\boldsymbol{M} \pm \boldsymbol{S E}[\mathbf{9 5 \%} \boldsymbol{C I}]$ \\
\hline Vocalization & $25.51 \pm 5.78[14.70-36.52]$ \\
Finger tapping & $59.18 \pm 6.63[46.32-72.36]$
\end{tabular}

$t(29)=4.50, p<0.001$

1101 This analysis was done on all go asynchronies, this means 1st go after stop signals, 2nd go after stop signals and all

1102 other go after stop signals. The analysis presented in the main text corresponded to only go asynchronies of 2 nd go

1103 after stop signals and all other go after stop signals. We decided to include this analysis to show that the exclusion of

1104 the 1st go after stop signals did no change the results. 


\section{Appendix H. Descriptive statistics}

1106

1107 Table A8. Descriptive statistics of go asynchronies, stop-signal delay (SSD), stop-signal 1108 reaction times (SSRTs) and go misses.

\begin{tabular}{lrr}
\hline \multicolumn{1}{c}{ Variables } & \multicolumn{1}{c}{$\begin{array}{c}\text { Vocalization } \\
\boldsymbol{M} \pm \boldsymbol{S E}[\mathbf{9 5 \%} \boldsymbol{C I}]\end{array}$} & \multicolumn{1}{c}{$\begin{array}{c}\text { Finger tapping } \\
\boldsymbol{M} \pm \boldsymbol{S E}[\mathbf{9 5 \%} \boldsymbol{C} \boldsymbol{]}]\end{array}$} \\
\hline Go-asynchrony_relevant stop & $-35.37 \pm 7.16[-50.01$ to -20.74$]$ & $-70.24 \pm 8.62[-87.86$ to -52.62$]$ \\
Go-asynchrony_irrelevant stop & $-61.47 \pm 5.92[-73.57$ to -49.37$]$ & $-128.29 \pm 9.19[-147.07$ to -109.51$]$ \\
SSD_relevant stop $_{\text {SSRT_relevant stop }}$ & $207.15 \pm 8.01[190.75$ to 223.54$]$ & $176.82 \pm 10.81[154.70$ to 198.93$]$ \\
Go misses_relevant stop + & $187.81 \pm 9.52[170.74$ to 205.56$]$ & $152.95 \pm 6.60[140.46$ to 156.60$]$ \\
Go misses_irelevant stop + & $1 \%$ & $3 \%$ \\
\hline
\end{tabular}

$1109 \mathrm{M}=$ mean in milliseconds

$1110 \quad \mathrm{SE}=$ Standard error

$111195 \% \mathrm{CI}=95 \%$ confidence intervals

$1112+$ This is the percentage of misses

1113 


\section{Appendix I. Checking assumptions of the stop signal task}

1115

1116 The stop signal task is based on a model called the horse race model developed by Logan and

1117 Cowan (1984). This model assumes independence of go and stop processes because failed stop

1118 RTs occur earlier than go-RTs. A finding that suggests in a failed stop trial, a response is given

1119 because the go process won the race against the stop process. In our current study, we did not

1120 have RTs but go asynchronies. We assume that in a failed stop trial, a response is given if the go

1121 process won the race against the stop, this would mean that the failed stop asynchrony would

1122 occur earlier compared to the go asynchrony. To check this assumption, we carried out a $2 \times 2$

1123 repeated measures ANOVA with the within-subject factors of 2 go categories (go

1124 asynchrony_relevant stop, failed stop asynchrony_relevant stop) and 2 response modalities (finger

1125 tapping and vocalization).

1126

1127 The results show that the main effects of both factors were statistically significant but not the

1128 interaction between these two factors. The go category factor $\left(F(1,29)=62.20, p<0.001, \eta^{2}{ }_{p}=\right.$

$11290.69)$ revealed that indeed the failed stop asynchrony_relevant stop $(M=-89.97, S E=6.84,95 \% C I=$

1130 [-103.94 to -75.99]) occurred significantly earlier compared to go asynchrony_relevant stop $(M=$ -

$113152.81, S E=7.28,95 \% C I=[-67.69$ to -37.92$])$. A finding that meets the horse race model

1132 assumption, suggesting the go process won the race against the stop process in the failed stop

1133 SR.

1134

1135 Moreover, the response modality factor $\left(F(1,29)=52.12, p<0.001, \eta^{2}{ }_{p}=0.65\right)$ showed that

1136 asynchronies of finger tapping $(M=-91.76, S E=7.78,95 \% C I=[-107.66$ to -75.86$])$ occurred

1137 significantly earlier compared to those asynchronies of vocalization $(M=-51.01, S E=6.64,95 \%$

$1138 C I=[-64.59$ to -37.44$])$. 